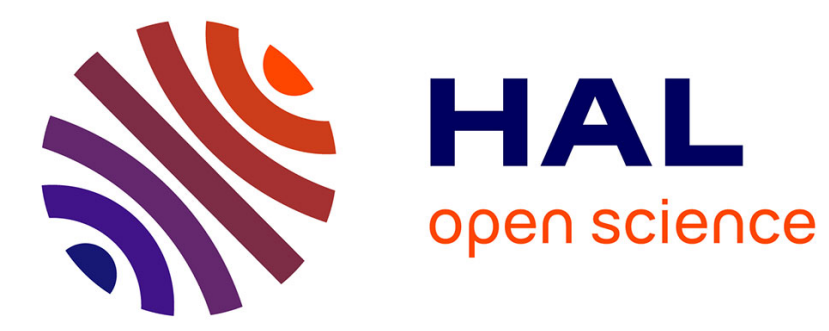

\title{
Shape optimization of an imperfect interface: steady-state heat diffusion
}

Grégoire Allaire, Beniamin Bogosel, Matias Godoy

\section{To cite this version:}

Grégoire Allaire, Beniamin Bogosel, Matias Godoy. Shape optimization of an imperfect interface: steady-state heat diffusion. 2021. hal-03102760v2

\section{HAL Id: hal-03102760 \\ https://hal.science/hal-03102760v2}

Preprint submitted on 13 Aug 2021

HAL is a multi-disciplinary open access archive for the deposit and dissemination of scientific research documents, whether they are published or not. The documents may come from teaching and research institutions in France or abroad, or from public or private research centers.
L'archive ouverte pluridisciplinaire HAL, est destinée au dépôt et à la diffusion de documents scientifiques de niveau recherche, publiés ou non, émanant des établissements d'enseignement et de recherche français ou étrangers, des laboratoires publics ou privés. 


\title{
Shape optimization of an imperfect interface: steady-state heat diffusion
}

\author{
Grégoire Allaire, Beniamin Bogosel and Matías Godoy C. ${ }^{1}$ \\ ${ }^{1}$ CMAP, CNRS, Ecole Polytechnique, Institut Polytechnique de Paris, \\ 91128 Palaiseau, France. matias.godoy@polytechnique.edu
}

\begin{abstract}
In the context of a diffusion equation, this work is devoted to a two-phase optimal design problem where the interface, separating the phases, is imperfect, meaning that the solution is discontinuous while the normal flux is continuous and proportional to the jump of the solution. The shape derivative of an objective function with respect to the interface position is computed by the adjoint method. Numerical experiments are performed with the level set method and an exact remeshing algorithm so that the interface is captured by the mesh at each optimization iteration. Comparisons with a perfect interface are discussed in the setting of optimal design or inverse problems.
\end{abstract}

Keywords: imperfect interfaces, shape optimization, level set method

\section{Introduction}

The problem of optimal distribution of materials or phases for a given physical system has a great relevance in the field of optimal design. The aim is to find the optimal distribution of materials, with prescribed proportions, such that a cost or performance criterion, which depends on the phases mixture, is minimized. This problem has been explored for several governing systems (for example in the scalar setting, for stationary heat, electrical or magnetic diffusion [47, 15], or in the vector case for linear elasticity $[10,5]$ ) with different techniques, such as the Hadamard method of shape variation [34], homogenization methods $[2,49]$ or the SIMP method $[13,51]$. In most works, the interface between phases is assumed to carry the classical transmission conditions, namely continuity across the interface of the solution and its normal flux, which in this work will be referred to as a perfect interface.

In this work, we rather focus on systems where this classical transmission condition between phases does not hold and we call it an imperfect interface. Such a behaviour is the result of imperfect interactions or bondings between materials and is taken into account in many works where this phenomenon has relevant and important physical consequences. For example, [43] studied the reinforcement of composite structures for compliance minimization when there is a competition between the rigidity of the solid inclusions and the imperfect interfacial bonding between the inclusions and the surrounding matrix. In [15] so-called latent heat storage systems are considered: they are storage devices to solve 
the mismatch between energy demand and supply. They are made of a mixture of high conductivity foams with so-called phase changing materials (PCM) which can store high volumes of heat per unit volume at the cost of having very low conductivity. Due to the big difference on material properties and the poor bonding due to material imperfections on the considered mixture, the resulting system features a heat loss on the interface between phases. Finally, and this is our true ultimate motivation, in metallic additive manufacturing structures are often built with the help of supports which are removed after completion of the manufacturing process [12], [32], [31]. To facilitate their removal, those supports are often weakly connected to the main structure by means of a jagged or indented layer, which can be modeled, at first order, as an imperfect interface between the supports and the structure. Optimization of such supports is a very active field, see [4] and references therein.

Although some motivating examples are in the setting of linear elasticity, the present work focus on the optimization of imperfect interfaces for the scalar model of heat diffusion. The extension of our results to the elasticity system case is the topic of a further work. Our model of imperfect interface is classical: the temperature is discontinuous through the interface but the normal heat flux is continuous and proportional to the temperature jump with a positive proportionality coefficient, which is called the conductance of the interface (see Section 1 for more details). For the steady-state heat conduction model and for a general cost function, we use the Hadamard boundary variation method in order to perform a shape sensitivity analysis. As is well known in the case of a perfect interface $[37,14,47,5]$, the solution of the boundary value problem is not differentiable with respect to the interface in the usual sense. Nevertheless, the transported solution, in a Lagrangian framework, is indeed differentiable with respect to the interface. It turns out to be enough for computing the shape (or interface) derivative of the cost function, using the Céa fast derivation method [18]. The same applies for an imperfect interface but the method is even more straightforward since the Lagrangian for Céa's method is simpler, not having to take into account the continuity of the solution at the interface. Once the expressions of shape derivatives are obtained, numerical interface optimization can be performed. To do so, we rely on a level set based mesh evolution method (as presented in [6]) in order to track and exactly mesh the interface. This is crucial because the imperfect interface condition leads to integral terms in the variational formulation which are carried by the interface. If a fixed mesh were used, it would require some interpolation process or a smoothed-interface model like in $[5,50]$. Clearly, resorting to an exact meshing of the interface avoids many further numerical errors and guarantees an accurate modeling during the optimization process.

As pointed out in previous works (see [15] and references therein), using continuous finite elements does not allow to evaluate discontinuous quantities properly, notably the jumps across interfaces. Rather, it requires to consider more ad-hoc techniques such as extended finite element methods (XFEM, see [28] for example). Since the interface is exactly meshed, we propose a simpler but effective method to compute the solution which is discontinuous through the interface. As explained in Section 5 we define two fields in the entire domain: each one correspond to the solution in one phase, but not in the other. A small penalization term in the other phase for each field allows to obtain a well-posed coupled system which approximates the exact equation.

The paper is organized as follows. In Section 1, the model and the optimization problem are presented. Section 2 discusses the functional framework for the governing system and some results of interest. Section 3 is devoted to the shape-sensitivity analysis, 
obtaining expressions for the shape derivative for a general class of objective functions. In Section 4 we briefly recall the level set method in our context, namely to perform a mesh evolution to track efficiently the interface. Finally Section 5 gives the implementation details and the optimization algorithm, while Section 6 contains our 2-d numerical experiments which are motivated by optimal design or by inverse problems.

\section{Problem Formulation}

We consider a working domain $D \subset \mathbb{R}^{d}$ with $d=2,3$, which is a smooth bounded open set, divided in two open subsets $\Omega_{1}$ and $\Omega_{2}=D \backslash \bar{\Omega}_{1}$, with piecewise- $\mathcal{C}^{2}$ regularity. The subsets $\Omega_{1}$ and $\Omega_{2}$ are refered as phases since different material properties hold inside. The phases are separated by a smooth interface $\Gamma$ which satisfies the following assumption.

Assumption 1.1. The interface $\Gamma$ is smooth and is described by one of the two different cases. In the first case, $\Gamma$ is a surface without boundary, included in $D$, and it holds $\Gamma=\partial \Omega_{1}$ with $\Gamma \cap \partial D=\emptyset\left(\Omega_{1}\right.$ can be seen as the interior or immersed phase and $\Omega_{2}$ as the exterior phase, with respect to $\Gamma$ ). In the second case, $\Gamma$ is a surface with a boundary $\partial \Gamma$ which is included in the exterior boundary, $\partial \Gamma \subset \partial D$, meaning that both phases touch $\partial D$ (see Figure 1 for examples of the two possible configurations).

Remark 1.2. A third case is not covered by our analysis, that of a surface $\Gamma$ with a boundary $\partial \Gamma$ which is included in $D$ but does not touch the exterior boundary $\partial D$. Of course, in such a case, it cannot be an interface separating two different phases. But it could be seen as a crack inside a single phase.

We consider a function $u$ defined in $D$ such that the restrictions to $\Omega_{1}$ and $\Omega_{2}$, denoted by $u_{1}$ and $u_{2}$ respectively, satisfy $[u]:=u_{2}-u_{1} \neq 0$ on $\Gamma$, i.e. $u$ is allowed to be discontinuous across the interface $\Gamma$. This function $u$ is the solution of the governing system:

$$
\begin{cases}-\operatorname{div}\left(A \nabla u_{i}\right)=f & \text { in } \Omega_{i} \\ \left(A \nabla u_{1}\right) \cdot n_{1}=\left(A \nabla u_{2}\right) \cdot n_{1} & \text { on } \Gamma \\ \left(A \nabla u_{1}\right) \cdot n_{1}=\alpha\left(u_{2}-u_{1}\right)=\alpha[u] & \text { on } \Gamma \\ A \nabla u \cdot n=g_{N} & \text { on } \Gamma_{N} \subset \partial D \\ u=g_{D} & \text { on } \Gamma_{D} \subset \partial D\end{cases}
$$

where $A(x)=A_{1}(x) \chi_{1}(x)+A_{2}(x) \chi_{2}(x), \chi_{i}$ is the characteristic function of the set $\Omega_{i}, i=$ $1,2, A_{1}, A_{2} \in C^{1}(\bar{D})$ are the conductivities in each phase with $\inf _{x \in \bar{D}} A_{i}>0$ and possibly $A_{2}(x)-A_{1}(x) \neq 0$ on $\Gamma, \alpha \in C^{1}(\bar{D})$ is the so-called conductance of the interface $\Gamma$ and is such that $\min _{x \in \bar{D}} \alpha>0,[u]=u_{2}-u_{1}$ is the jump of $u$ across the interface $\Gamma, n_{1}$ is the exterior normal of $\Omega_{1}, n$ is the exterior normal to $D, \partial D=\Gamma_{D} \cup \Gamma_{N}$ with $\Gamma_{D} \neq \emptyset$ and $f \in H^{1}(D), g_{D} \in H^{1 / 2}\left(\Gamma_{D}\right), g_{N} \in L^{2}(\partial D)$ are given (see the next section for a statement about existence of solutions to (1.1)).

Our aim is to determine an optimal distribution for $\Omega_{1}$ and $\Omega_{2}$, or equivalently, we want to find the optimal location for the interface $\Gamma$, such that the following cost functional is minimized:

$$
J(\Gamma)=\int_{D} j(u) d x+\int_{\Gamma_{N}} k(u) d s
$$


where $u \equiv u(\Gamma)$ is the unique solution of (1.1), $j$ and $k$ are smooth functions satisfying quadratic growth conditions. In order to avoid 'obvious configurations' on the minimization of $J$ (e.g. filling completely with the 'best' material with respect to the functional) a volume constraint for the phase $\Omega_{1}$ is added.
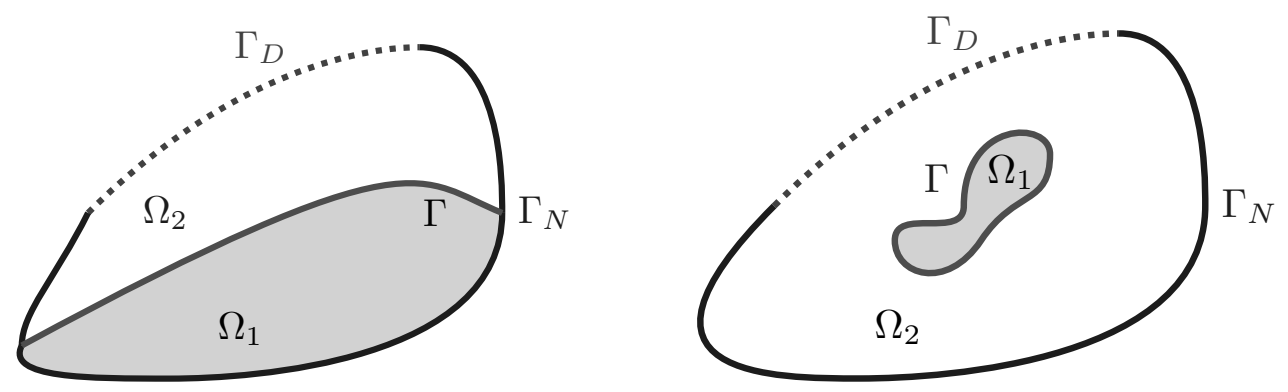

Figure 1: Two possible configurations of the interface $\Gamma$.

With this, our optimization problem reads:

$$
\begin{array}{r}
\min _{\Gamma \in \mathcal{U}_{a d}} J(\Gamma) \\
\text { s.t. } \int_{\Omega_{1}} d x=V_{\text {tar }},
\end{array}
$$

where $\mathcal{U}_{a d}$ is a set of admissible interfaces (for example, uniformly smooth) and $V_{t a r}$ is a target volume for the phase $\Omega_{1}$.

As mentioned in the introduction, the governing system can be seen as a stationary thermal conduction between two different materials with conductivities $A_{1}, A_{2}$, where the interface $\Gamma$ between them has a resistance, which is due to poor mechanical and chemical bounds between the phases. This resistance induces a heat loss, modeled by a discontinuity of the solution across $\Gamma$ and a Fourier type law for the continuous normal flux through $\Gamma$ (see $[15,48]$ for a more detailed explanaiton of this phenomenon). In the context of elasticity, in [42], imperfect bonding or partial adhesion is described as the result of interfacial damage due to service or as a consequence of the surface properties of the fiber and matrix materials. In any case, this is represented as a discontinuity of the solution for the considered governing system across the interface. A more detailed deduction of this type of interfacial conditions can be found in $[1,40]$.

The well-posedness of the optimal design problem is not discussed here. However, as explained in several works (see $[11,19,20]$ ), existence of optimal designs for this type of problems usually requires to include in the definition of $\mathcal{U}_{a d}$ some uniform smoothness, or geometrical or topological constraints.

Remark 1.3. We recall that the outer boundary $\partial D$ and its subsets, where boundary conditions hold, are not allowed to move (for example, due to design reasons). The only optimization variable is the interface $\Gamma$.

Notation 1.4. In order to avoid confusion between the different normals (to the domain $D$, or to the interface $\Gamma$ ), the normal to the interaface $\Gamma$ is denoted by $\nu=n_{1}=-n_{2}$, where $n_{i}$ stands for the exterior normal with respect to $\Omega_{i}$, and the notation $n$ is reserved for the exterior normal to the domain $D$. 


\section{Review of Broken Sobolev Spaces}

In order to study problem (1.1), we introduce so-called broken Sobolev spaces, where the functions are allowed to be discontinuous at the interface between phases. These spaces are also relevant in the context of discontinuous Galerkin methods (dG) in numerical analysis. In order to simplify the presentation, we present these spaces and their results in our particular context (the more interested reader is invited to study the following references [25, 27], where the treatment is general).

Given a smooth bounded domain $D$ we denote by $L^{p}(D)$ with $p>0$ and $H^{s}(D)$ with $s>0$ the usual Lebesgue and Sobolev spaces. Also, given a relatively open part of the boundary $\Gamma_{D} \subset \partial D$ with non-zero $(d-1)$-dimensional measure, we consider the space:

$$
H_{\Gamma_{D}}^{1}(D):=\left\{v \in H^{1}(D), v=0 \text { on } \Gamma_{D}\right\} .
$$

Now, suppose that $D$ is such that $\bar{D}=\bar{\Omega}_{1} \cup \bar{\Omega}_{2}$, where $\Omega_{1}$ and $\Omega_{2}$ are disjoint open sets, $\Omega_{1} \cap \Omega_{2}=\emptyset$, which are separated by an interface $\Gamma=\partial \Omega_{1} \cap \partial \Omega_{2}$, the broken space $H_{b}^{1}\left(D ; \Omega_{1}, \Omega_{2}\right)$ is defined by

$$
H_{b}^{1}\left(D ; \Omega_{1}, \Omega_{2}\right):=\left\{u \in L^{2}(D) \text { such that } u_{1}:=\left.u\right|_{\Omega_{1}} \in H^{1}\left(\Omega_{1}\right), u_{2}:=\left.u\right|_{\Omega_{2}} \in H^{1}\left(\Omega_{2}\right)\right\} .
$$

We simply write $H_{b}^{1}$ for this space, except when explicit mention of the partition of $D$ is needed. This space is endowed with the so-called broken norm:

$$
\|u\|_{H_{b}^{1}}:=\left(\left\|u_{1}\right\|_{H^{1}\left(\Omega_{1}\right)}^{2}+\left\|u_{2}\right\|_{H^{1}\left(\Omega_{2}\right)}^{2}\right)^{1 / 2}
$$

with this norm, $H_{b}^{1}$ is a Hilbert space. From the definition of $H_{b}^{1}$ is clear that $H^{1}(D) \subset$ $H_{b}^{1}$ and in general the inclusion is strict, as we allow the function in $H_{b}^{1}$ to have jump discontinuities along $\Gamma$. In fact, the following result (see [25]) states a natural condition for functions in $H_{b}^{1}$ to be in $H^{1}(D)$.

Lemma 2.1. A function $v \in H_{b}^{1}\left(D ; \Omega_{1}, \Omega_{2}\right)$ belongs to $H^{1}(D)$ if and only if

$$
[v]:=\left.v\right|_{\Omega_{2}}-\left.v\right|_{\Omega_{1}}=0 \text { on } \Gamma \text {. }
$$

We recall a result of [15] for the well-posedness of problem (1.1) (actually, the proof in [15] focuses on the case when $\Gamma$ is a closed surface but the extension to the general case follows the same arguments). Define the subspace

$$
H_{b, 0}^{1}:=\left\{v \in H_{b}^{1}: v=0 \text { on } \Gamma_{D}\right\} .
$$

Then, (1.1) admits the following variational formulation

$$
\left\{\begin{array}{l}
\text { Find } u \in H_{b}^{1}, \text { such that } u=g_{D} \text { on } \Gamma_{D} \text { and, for all } v \in H_{b, 0}^{1}, \\
\int_{\Omega_{1}} A_{1} \nabla u_{1} \cdot \nabla v_{1}, d x+\int_{\Omega_{2}} A_{2} \nabla u_{2} \cdot \nabla v_{2} d x+\int_{\Gamma} \alpha[u][v] d s=\int_{D} f v d x+\int_{\Gamma_{N}} g_{N} v d s .
\end{array}\right.
$$

For this variational problem, we have the following result.

Theorem 2.2. For any data $f \in L^{2}(D), g_{D} \in H^{1 / 2}\left(\Gamma_{D}\right), g_{N} \in L^{2}(\partial D)$, the problem (1.1) or (2.4) has a unique solution $u \in H_{b}^{1}$. This solution satisfies

$$
\|u\|_{H_{b}^{1}} \leq C\left(\|f\|_{L^{2}(D)}+\left\|g_{D}\right\|_{H^{1 / 2}\left(\Gamma_{D}\right)}+\left\|g_{N}\right\|_{L^{2}\left(\Gamma_{N}\right)}\right) .
$$


Assumption 2.3. In the following, we assume that the solution $u$ of system (1.1) (or that of (2.6)) is smooth in each phase, namely $u_{i}=\left.u\right|_{\Omega_{i}} \in H^{2}\left(\Omega_{i}\right)$. This assumption can be proved to hold true if the data $f, g_{N}, g_{D}$ are smooth, as well as the interface $\Gamma$. The only difficulty comes from the possible interface between $\Gamma_{D}$ and $\Gamma_{N}$. Indeed, it is well known that at points in $\bar{\Gamma}_{D} \cap \bar{\Gamma}_{N}$ the solution u may feature a singularity such that it does not belong to $H^{2}\left(\Omega_{i}\right)$. We exclude this possibility which creates technical difficulties which are not at the core of our study. It does not happen, for example, if we assume that $\Gamma_{D}$ and $\Gamma_{N}$ are two different connected components of $\partial D$.

Notice that, when $\alpha$ is a constant, it can be seen as a penalization parameter for the jump $[u]$. So, if we let $\alpha \rightarrow+\infty$, it is expected that $[u]$ goes to zero and therefore, in this limit, a perfect transmission condition is recovered at the interface $\Gamma$. The following result formalizes this idea.

Lemma 2.4. Denote by $u^{\alpha}$ the solution of (2.4) for a given $\alpha>0$. As a goes to $+\infty$ the sequence $u^{\alpha}$ converges strongly for the norm of $H_{b}^{1}\left(D ; \Omega_{1}, \Omega_{2}\right)$ to a limit $u^{*} \in H^{1}(D)$, which is the solution of the following boundary value problem, which is referred as the 'perfect interface' problem:

$$
\begin{cases}-\operatorname{div}\left(A \nabla u_{i}\right)=f & \text { in } \Omega_{i} \\ u_{1}=u_{2} & \text { on } \Gamma \\ A_{1} \nabla u_{1} \cdot \nu=A_{2} \nabla u_{2} \cdot \nu & \text { on } \Gamma \\ A \nabla u \cdot n=g_{N} & \text { on } \Gamma_{N} \\ u=g_{D} & \text { on } \Gamma_{D}\end{cases}
$$

Proof. Without loss of generality, we can take $g_{D}=0$, so that $u^{\alpha} \in H_{b, 0}^{1}$. From Theorem 2.2 and using the variational formulation (2.4) with $v=u^{\alpha}$ we have:

$$
\left\|u^{\alpha}\right\|_{H_{b}^{1}} \leq C \quad \text { and } \quad\left\|\left[u^{\alpha}\right]\right\|_{L^{2}(\Gamma)} \leq \frac{C}{\sqrt{\alpha}}
$$

with $C$ independent of $\alpha$. Letting $\alpha \rightarrow+\infty$ we deduce from the first inequality the existence of a subsequence and a limit $u^{*} \in H_{b, 0}^{1}$ such that $u^{\alpha}$ converges weakly to $u^{*}$ in $H_{b, 0}^{1}$. In particular, introducing $u_{i}^{*}=\left.u^{*}\right|_{\Omega_{i}} \in H^{1}\left(\Omega_{i}\right), u_{i}^{\alpha}$ converges weakly to $u_{i}^{*}$ in $H^{1}\left(\Omega_{i}\right)$. From the second inequality we deduce that the jump $\left[u^{\alpha}\right]$ converges strongly to zero in $L^{2}(\Gamma)$ and, by the trace theorem, that the jump of the limit vanishes, $\left[u^{*}\right]=0$. Thus, from Lemma (2.1), it implies $u^{*} \in H^{1}(D)$. Now, taking $v \in H_{\Gamma_{D}}^{1}(D)$ as a test function in the variational formulation (2.4) and passing to the limit we obtain that $u^{*}$ solves (2.6). For the strong convergence it suffices to recognize that, for the test function $v=u^{\alpha}$, the linear form of the variational formulation (2.4) converges and to use the lower semi-continuity of the bilinear form. In particular it implies that $\sqrt{\alpha}\left[u^{\alpha}\right]$ converges strongly to 0 in $L^{2}(\Gamma)$.

\section{Shape-sensitivity analysis}

As the goal is to determine the optimal location of the interface $\Gamma$ in order to minimize (1.2), we rely on the concept of shape derivatives, based on the Hadamard boundary variation method (see [36]). More precisely, the interface $\Gamma$ is perturbed by a vector field $\theta \in W^{1, \infty}\left(\mathbb{R}^{d}, \mathbb{R}^{d}\right)$ in the following sense:

$$
\theta \mapsto \Gamma_{\theta}:=(I d+\theta)(\Gamma) \quad \text { or } \quad \theta \mapsto \Omega_{1, \theta}:=(I d+\theta)\left(\Omega_{1}\right)
$$


where $(I d+\theta)(\Gamma):=\{x+\theta(x): x \in \Gamma\}$. Because $\Gamma$ is restricted to belong to $D$, we introduce the following set of admissible deformations $\Theta_{a d}$

$$
\Theta_{a d}:=\left\{\theta \in W^{1, \infty}\left(D, \mathbb{R}^{d}\right):\|\theta\|_{W^{1, \infty}}<1, \theta \cdot n=0 \text { on } \partial D\right\} .
$$

Note in passing that $\Theta_{a d}$ could be used to define a possible set of admissible interfaces: given a reference smooth interface $\Gamma^{0}$, all possible interfaces are perturbation of $\Gamma^{0}$ by an admissible deformation $\theta \in \Theta_{a d}$, namely

$$
\mathcal{U}_{a d}:=\left\{\Gamma \subset D: \exists \theta \in \Theta_{a d} \text { such that } \Gamma=(I d+\theta)\left(\Gamma^{0}\right)\right\} .
$$

With this, we can define the concept of shape derivative of a functional.

Definition 3.1. A function $J(\Gamma)$ will be shape differentiable at $\Gamma$ if the map $\theta \in \Theta_{a d} \mapsto$ $J\left(\Gamma_{\theta}\right)$ is Fréchet-differentiable at 0 . The corresponding derivative is denoted by $J^{\prime}(\Gamma) \in$ $W^{1, \infty}\left(D, \mathbb{R}^{d}\right)^{*}$ and the following asymptotic expansion holds in a neighborhood of $0 \in$ $W^{1, \infty}\left(D, \mathbb{R}^{d}\right)$ :

$$
J\left(\Gamma_{\theta}\right)=J(\Gamma)+\left\langle J^{\prime}(\Gamma), \theta\right\rangle+o(\theta) \text {, with } \lim _{\theta \rightarrow 0} \frac{|o(\theta)|}{\|\theta\|_{W^{1, \infty}}}=0 .
$$

The Hadamard method is widely used to determine derivatives with respect to single phase problems, or shape optimization problems (see for example the textbooks $[3,36$, 23]). In an interface context there are fewer works: we mention for the scalar case $[37,47,14]$, and in an elasticity context in $[5,10,8,38]$. Finally, we can consider multiphysics problems, where not only several phases are considered, but also on each one there are different physical phenomena, see for example [29] for an in-depth analysis of several problems of this type. It is worth to mention that in all these works the interfaces are assumed to be perfect, this is, there are no discontinuities allowed across them.

In the following, we compute the shape derivatives using the Céa fast derivation method [18]. We recall the results for a perfect interface setting and we present new results in the imperfect interface setting. We first recall a classical but essential result regarding the shape derivation of costs functionals where fixed functions are integrated on a perturbed domain:

Lemma 3.2. Let $\mathcal{O}$ be a smooth bounded open set of $\mathbb{R}^{d}$ and $\theta \in W^{1, \infty}\left(\mathbb{R}^{d}, \mathbb{R}^{d}\right)$. Let $f \in H^{1}\left(\mathbb{R}^{d}\right)$ and $g \in H^{2}\left(\mathbb{R}^{d}\right)$ be two given functions. Assume that $\gamma$ is a smooth subset of $\partial \mathcal{O}$ with boundary $\partial \gamma$. Then, the shape derivatives of

$$
J_{1}(\mathcal{O})=\int_{\mathcal{O}} f d x \text { and } J_{2}(\gamma)=\int_{\gamma} g d s
$$

are given by:

$$
\left\langle J_{1}^{\prime}(\mathcal{O}), \theta\right\rangle=\int_{\partial \mathcal{O}} f \theta \cdot \nu d s
$$

and

$$
\left\langle J_{2}^{\prime}(\gamma), \theta\right\rangle=\int_{\gamma}\left(\frac{\partial g}{\partial \nu}+g \kappa\right) \theta \cdot \nu d s+\int_{\partial \gamma} g \theta \cdot \tau d l,
$$

respectively, where $\nu$ is the unit normal vector to $\partial \mathcal{O}, \kappa$ is the mean curvature and $\tau$ is the unit tangent vector to $\partial \mathcal{O}$ such that $\tau$ is normal to both $\partial \gamma$ and $\nu$, and $d l$ is the $(d-2)$ dimensional measure along $\partial \gamma$.

Remark 3.3. The result (3.3) is usually stated when $\gamma=\partial \mathcal{O}$ (see [36]) and thus has no boundary $\partial \gamma$. When $\gamma$ has a boundary, the proof of (3.3) yields an additional term on $\partial \gamma$ which is due to an integration by parts on the surface $\gamma$ (see [39]). 


\subsection{Perfect bonding}

In this context, the state equation is (2.6) and we define the adjoint problem:

$$
\begin{cases}-\operatorname{div}\left(A \nabla p_{i}\right)=-j^{\prime}\left(u_{i}\right) & \text { in } \Omega_{i} \\ p_{1}=p_{2} & \text { on } \Gamma \\ A_{1} \nabla p_{1} \cdot \nu=A_{2} \nabla p_{2} \cdot \nu & \text { on } \Gamma \\ p=0 & \text { on } \Gamma_{D} \\ A \nabla p \cdot n=-k^{\prime}(u) & \text { on } \Gamma_{N}\end{cases}
$$

Due to the smoothness and growth conditions given on $f, g_{D}, g_{N}, j$ and $k$, the problems (2.6) and (3.4) are well posed and their unique solutions satisfy $u, p \in H^{1}(D)$. According to Assumption 2.3 the restriction of $u$ in each phase is smooth, namely $u_{1} \in H^{2}\left(\Omega_{1}\right), u_{2} \in$ $H^{2}\left(\Omega_{2}\right)$. In the sequel, the same smoothness assumption is made on the adjoint $p$. The computation of the shape derivative for (1.2) is well known [47].

Theorem 3.4. Under Assumptions 1.1 and 2.3, the function $J$ is shape differentiable with respect to variations of the interface $\Gamma$, and we have, for $\theta \in \Theta_{a d}$ :

$$
\left\langle J^{\prime}(\Gamma), \theta\right\rangle=-\int_{\Gamma}[A]\left(\partial_{\tau} u\right)\left(\partial_{\tau} p\right) \theta \cdot \nu d s+\int_{\Gamma}\left[A^{-1}\right](A \nabla u \cdot \nu)(A \nabla p \cdot \nu) \theta \cdot \nu d s,
$$

where $u$ solves (2.6), p solves (3.4), the jump [.] is defined as $[\varphi]=\varphi_{2}-\varphi_{1}, \nu=n_{1}=-n_{2}$ and $\partial_{\tau} \phi=\nabla \phi-(\nabla \phi \cdot \nu) \nu$.

It is remarkable that formula (3.5) depends explicitly on the jumps of physical properties. Obviously, if $A_{1}=A_{2}$ on $\Gamma$, then $\left\langle J^{\prime}(\Gamma), \theta\right\rangle=0$ which makes sense because in that case the materials have the same properties and therefore a motion of the interface does not imply any improvement of the cost functional.

Remark 3.5. The formula (3.5) is presented with the opposite sign with respect to [47]. This is due to the sign chosen for the right hand side in the adjoint problem (3.4).

Proof. Fort the sake of completeness, we recall the main idea for the actual computation of the shape derivative of $J$, which relies on Céa's fast derivation method [18]. A complete proof can be found in [47] (see also [7]). The key is to introduce a Lagrangian which separates the two subdomains $\Omega_{1}, \Omega_{2}$ and takes care of the transmission conditions on $\Gamma$ (this issue will be different for the imperfect interface case). The reason is that the solution of (2.6) is smooth in each subdomain but not globally in $D$. Consider the Lagrangian $\mathcal{L}: \Theta_{a d} \times H^{1}(D) \times H^{1}(D) \times H_{\Gamma_{D}}^{1}(D) \times H_{\Gamma_{D}}^{1}(D) \times H^{1}(D) \times H^{1}(D) \rightarrow \mathbb{R}$ given by:

$$
\begin{aligned}
\mathcal{L}\left(\theta, v_{1}, v_{2}, q_{1}, q_{2}, \lambda_{1}, \lambda_{2}\right) & :=\sum_{i=1}^{2} \int_{(I+\theta) \Omega_{i}} j\left(v_{i}\right) d x+\int_{(I+\theta) \Omega_{i}} A_{i} \nabla v_{i} \cdot \nabla q_{i} d x-\int_{(I+\theta) \Omega_{i}} f q_{i} d x \\
& -\int_{\Gamma_{N} \cap \partial \Omega_{i}} g_{N} \cdot q_{i} d s+\frac{1}{2} \int_{(I+\theta) \Gamma}\left(A_{1} \partial_{\nu} q_{1}+A_{2} \partial_{\nu} q_{2}\right) \cdot\left(v_{2}-v_{1}\right) d s \\
& +\frac{1}{2} \int_{(I+\theta) \Gamma}\left(A_{1} \partial_{\nu} v_{1}+A_{2} \partial_{\nu} v_{2}\right) \cdot\left(q_{2}-q_{1}\right) d s+\sum_{i=1}^{2} \int_{\Gamma_{N} \cap \partial \Omega_{i}} k\left(v_{i}\right) d s \\
& +\int_{\Gamma_{D} \cap \partial \Omega_{i}} \lambda_{i}\left(v_{i}-g_{D}\right) d s .
\end{aligned}
$$


As usual, differentiating $\mathcal{L}$ with respect to $q_{1}, q_{2}, \lambda_{1}, \lambda_{2}$ and setting to 0 the obtained derivatives evaluated at $v_{1}=u_{1}, v_{2}=u_{2}$ provide the state equation (2.6). An analogous argument, differentiating with respect to $v_{1}, v_{2}$, provides the adjoint system (3.4). Now, we get for any $q_{1}, q_{2}, \lambda_{1}, \lambda_{2}$ :

$$
\mathcal{L}\left(\theta, u_{1}, u_{2}, q_{1}, q_{2}, \lambda_{1}, \lambda_{2}\right)=J(\Gamma) .
$$

Then, using the chain rule lemma and imposing the previous optimality conditions we deduce:

$$
\left\langle J^{\prime}(\Gamma), \theta\right\rangle=\partial_{\theta} \mathcal{L}\left(0, u_{1}, u_{2}, p_{1}, p_{2}, \lambda_{1}\left(p_{1}\right), \lambda_{2}\left(p_{2}\right)\right)(\theta) .
$$

The derivative on the right hand side is computed using Lemma 3.2, decomposing the gradient in its normal and tangential part and imposing the transmission conditions satisfied by the state and adjoint solutions. Notice that no additional terms on $\partial \Gamma$ are present in (3.5) because of the transmission conditions.

Remark 3.6. An alternative expression for the shape derivative (3.5) is:

$$
\left\langle J^{\prime}(\Gamma), \theta\right\rangle=\int_{\Gamma}\left(A_{1} \nabla u_{1} \cdot \nabla p_{1}-A_{2} \nabla u_{2} \cdot \nabla p_{2}+2 A_{2} \partial_{\nu} u_{2} \partial_{\nu} p_{2}-2 A_{1} \partial_{\nu} u_{1} \partial_{\nu} p_{1}\right) \theta \cdot \nu d s .
$$

\section{$3.2 \quad$ Imperfect bonding}

In the case of imperfect bonding, we have to consider the lack of continuity for the solution of the state equation (1.1) on the interface $\Gamma$. We introduce the corresponding adjoint problem:

$$
\begin{cases}-\operatorname{div}\left(A \nabla p_{i}\right)=-j^{\prime}\left(u_{i}\right) & \text { in } \Omega_{i}, \\ A_{1} \nabla p_{1} \cdot \nu=A_{2} \nabla p_{2} \cdot \nu & \text { on } \Gamma, \\ A \nabla p \cdot \nu=\alpha\left(p_{2}-p_{1}\right) & \text { on } \Gamma, \\ p=0 & \text { on } \Gamma_{D}, \\ A \nabla p \cdot n=-k^{\prime}(u) & \text { on } \Gamma_{N},\end{cases}
$$

where $p$ is the adjoint state defined in $D$, with its restrictions to $\Omega_{1}$ and $\Omega_{2}$, denoted by $p_{1}$ and $p_{2}$. Thanks to the smoothness and growth conditions on $f, g_{D}, g_{N}, j$ and $k$, by Theorem 2.2 problems (1.1) and (3.7) are well posed in the broken space $H_{b}^{1}$, with unique solutions $u, p \in H_{b}^{1}$. Furthermore, according to Assumption 2.3 their restrictions on each phase satisfy $u_{1}, p_{1} \in H^{2}\left(\Omega_{1}\right), u_{2}, p_{2} \in H^{2}\left(\Omega_{2}\right)$.

Theorem 3.7. Under Assumptions 1.1 and 2.3, the shape derivative of the functional $J$, for $\theta \in \Theta_{a d}$ is given by:

$$
\begin{aligned}
\left\langle J^{\prime}(\Gamma), \theta\right\rangle=\int_{\Gamma}(-[j(u)]- & {\left.\left[A \partial_{\tau} u \cdot \partial_{\tau} p\right]+f[p]+\left(\alpha \kappa+\alpha^{2}\left[A^{-1}\right]+\partial_{\nu} \alpha\right)[u][p]\right) \theta \cdot \nu d s } \\
& +\int_{\partial \Gamma \cap \Gamma_{N}} \alpha[p][u] \theta \cdot \tau d l+\int_{\partial \Gamma \cap \Gamma_{N}}\left(g_{N}[p]-[k(u)]\right) \theta \cdot \boldsymbol{t} d l,
\end{aligned}
$$

where $u$ solves (1.1), $p$ solves (3.7), $\kappa$ stands for the mean curvature, the jump [.] is defined as $[\varphi]=\varphi_{2}-\varphi_{1}, \nu=n_{1}=-n_{2}, \tau$ is the unit tangent vector to $\Gamma$ which is normal to $\partial \Gamma, \boldsymbol{t}$ is the unit tangent vector to $\partial D$ which is normal to $\partial \Gamma$ and $d l$ is the $(d-2)$ dimensional measure along $\partial \Gamma \cap \Gamma_{N}$. 
Remark 3.8. The two unit vectors $\tau$ and $\boldsymbol{t}$ are different, the first one being tangent to $\Gamma$ while the second one is tangent to $\partial D$. The first one appears because of the variation of an integral on $\Gamma$, as studied in Lemma 3.2. The second one is a consequence of varying an integral on a subset of the fixed surface $\Gamma_{N}$.

Proof. According to Céa's method, we introduce the Lagrangian:

$$
\begin{aligned}
\mathcal{L}: \Theta_{a d} \times H^{1}(D) & \times H^{1}(D) \times H_{\Gamma_{D}}^{1}(D) \times H_{\Gamma_{D}}^{1}(D) \times H^{1}(D) \times H^{1}(D) \rightarrow \mathbb{R} \\
\mathcal{L}\left(\theta, v_{1}, v_{2}, q_{1}, q_{2}, \lambda_{1}, \lambda_{2}\right) & :=\sum_{i=1}^{2} \int_{(I+\theta) \Omega_{i}} j\left(v_{i}\right) d x+\int_{(I+\theta) \Omega_{i}} A_{i} \nabla v_{i} \cdot \nabla q_{i} d x-\int_{(I+\theta) \Omega_{i}} f q_{i} d x \\
& -\int_{\Gamma_{N} \cap \partial \Omega_{i}} g_{N} \cdot q_{i} d s+\int_{(I+\theta) \Gamma} \alpha\left(v_{2}-v_{1}\right)\left(q_{2}-q_{1}\right) d s \\
& +\sum_{i=1}^{2} \int_{\Gamma_{N} \cap \partial \Omega_{i}} k\left(v_{i}\right) d s+\int_{\Gamma_{D} \cap \partial \Omega_{i}} \lambda_{i}\left(v_{i}-g_{D}\right) d s .
\end{aligned}
$$

As usual, differentiating with respect to $v_{1}, v_{2}$ and imposing the optimality condition, lead to the adjoint problem (3.7). Differentiating with respect to $q_{1}, q_{2}, \lambda_{1}, \lambda_{2}$ and imposing the optimality condition, yield the state equation (1.1). Recognizing the variational formulation (2.4), the Lagrangian simplifies when $\left(v_{1}, v_{2}\right)=\left(u_{1}, u_{2}\right)$ and $\forall q_{1}, q_{2}, \lambda_{1}, \lambda_{2}$ we have:

$$
\mathcal{L}\left(\theta, u_{1}, u_{2}, q_{1}, q_{2}, \lambda_{1}, \lambda_{2}\right)=J(\Gamma) .
$$

Then, using the chain rule lemma and imposing the previous optimality conditions we deduce:

$$
\left\langle J^{\prime}(\Gamma), \theta\right\rangle=\partial_{\theta} \mathcal{L}\left(0, u_{1}, u_{2}, p_{1}, p_{2}, \lambda_{1}\left(p_{1}\right), \lambda_{2}\left(p_{2}\right)\right)(\theta),
$$

where the right-hand side has to be computed by using Lemma 3.2. As the functions $u_{1}, u_{2}, p_{1}, p_{2}$ are fixed when differentiating with respect to $\theta$ (we drop the reference to $\lambda_{1}, \lambda_{2}$ as their integrals vanishes when we evaluate at $\left.u_{1}, u_{2}\right)$, we obtain:

$$
\begin{aligned}
\partial_{\theta} \mathcal{L}\left(0, u_{1}, u_{2}, p_{1}, p_{2}\right)(\theta) & =\int_{\Gamma} \theta \cdot \nu\left(j\left(u_{1}\right)-j\left(u_{2}\right)\right) d s+\int_{\Gamma} \theta \cdot \nu\left(A_{1} \nabla u_{1} \cdot \nabla p_{1}-A_{2} \nabla u_{2} \cdot \nabla p_{2}\right) d s \\
& +\int_{\Gamma} \theta \cdot \nu\left(f p_{2}-f p_{1}+\kappa \alpha\left(u_{2}-u_{1}\right)\left(p_{2}-p_{1}\right)+\partial_{\nu}\left(\alpha\left(u_{2}-u_{1}\right)\left(p_{2}-p_{1}\right)\right)\right) d s \\
& +\int_{\partial \Gamma} \alpha[u][p] \theta \cdot \tau d l+\int_{\partial \Gamma \cap \Gamma_{N}}\left(g_{N}[p]-[k(u)]\right) \theta \cdot \boldsymbol{t} d l,
\end{aligned}
$$

where the two last integrals do not appear if $\Gamma$ has no boundary, $\partial \Gamma=\emptyset$. Because of the same Dirichlet boundary condition on $\Gamma_{D}$ for both phases, the penultimate integral on $\Gamma$ reduces to an integral on $\partial \Gamma \cap \Gamma_{N}$. The last integral features the unit vector $\boldsymbol{t}$ instead of $\tau$ because it comes from an application of Lemma 3.2 where the open set $\mathcal{O} \subset \mathbb{R}^{d}$ is replaced by $\Gamma_{N} \cap \partial \Omega_{i}$, a $(d-1)$-dimensional surface. Expressing the gradient in the local basis $(\tau, \nu)$ we have $\nabla u=\partial_{\tau} u \tau+\partial_{\nu} u \nu$, and then:

$$
\begin{aligned}
\partial_{\theta} \mathcal{L}\left(0, u_{1}, u_{2}, p_{1}, p_{2}\right)(\theta) & =\int_{\Gamma}(\theta \cdot \nu)\left(-[j(u)]-\left[A \partial_{\tau} u \partial_{\tau} p\right]+f[p]+[u][p]\left(\alpha \kappa+\partial_{\nu} \alpha\right)\right) d s \\
& +\int_{\Gamma}(\theta \cdot \nu)\left(A_{1} \partial_{\nu} u_{1} \partial_{\nu} p_{1}-A_{2} \partial_{\nu} u \partial_{\nu} p_{2}+\alpha \partial_{\nu}\left(u_{2}-u_{1}\right)[p]+\alpha \partial_{\nu}\left(p_{2}-p_{1}\right)[u]\right) d s \\
& +\int_{\partial \Gamma \cap \Gamma_{N}} \alpha[u][p] \theta \cdot \tau d l+\int_{\partial \Gamma \cap \Gamma_{N}}\left(g_{N}[p]-[k(u)]\right) \theta \cdot \boldsymbol{t} d l .
\end{aligned}
$$


To conclude we have to do some algebra:

$$
\begin{aligned}
A_{1} \partial_{\nu} u_{1} \partial_{\nu} p_{1}-A_{2} \partial_{\nu} u \partial_{\nu} p_{2} & +\alpha \partial_{\nu}\left(u_{2}-u_{1}\right)[p]+\alpha \partial_{\nu}\left(p_{2}-p_{1}\right)[u] \\
& =A \partial_{\nu} u \partial_{\nu}\left(p_{1}-p_{2}\right)+\partial_{\nu}\left(u_{2}-u_{1}\right) \alpha[p]+\partial_{\nu}\left(p_{2}-p_{1}\right) \alpha[u] \\
& =\partial_{\nu}\left(u_{2}-u_{1}\right) \alpha[p], \text { with, } \\
\partial_{\nu}\left(u_{2}-u_{1}\right)=\frac{1}{A_{2}} A_{2} \partial_{\nu} u_{2} & -\frac{1}{A_{1}} A_{1} \partial_{\nu} u_{1}=\frac{1}{A_{2}} \alpha[u]-\frac{1}{A_{1}} \alpha[u]=\alpha[u]\left(\frac{1}{A_{2}}-\frac{1}{A_{1}}\right)=\alpha[u]\left[A^{-1}\right],
\end{aligned}
$$

from which we deduce the desired formula.

Remark 3.9. The Lagrangian (3.9) has a simpler expression in comparison with the Lagrangian of the perfect interface case given by (3.6). This is due to the fact that in the first case the expression comes naturally from the variational formulation (2.4) of (1.1), while in the latter the variational formulation of (2.6) hides the transmission conditions on $\Gamma$, so they should be imposed in the Lagrangian via carefully chosen Lagrange multipliers.

Remark 3.10. Theorem 3.7 holds under the geometrical Assumption 1.1 on the interface $\Gamma$. One may wonder what happen in the case of a surface $\Gamma$ with a non-empty boundary $\partial \Gamma$ contained in the interior of $D$ (not touching $\partial D$ ), for which Assumption 1.1 is no longer valid. Of course, in such a case $\Gamma$ cannot be an interface separating two different phases. However, if the two phases are identical, $\Gamma$ can be interpreted as a fracture or a surface defect embedded in a uniform material. For such a configuration (identical phases and $\Gamma$ being a crack inside D), it is well known that the solution of (1.1) is usually singular around the crack tips, namely in the vicinity of $\partial \Gamma$. Thus, the smoothness Assumption 2.3 does not hold true and formula (3.8) is usually not valid. A correct shape derivative has been obtained in [30], [41] (see also [22] for a similar framework), to which we refer for further discussions and references. Nevertheless, if one insists in taking for granted the smoothness Assumption 2.3, then the proof of Theorem 3.7 is still valid and formula (3.8) simplifies because there is no last integral since $\partial \Gamma \cap \Gamma_{N}=\emptyset$ and the penultimate integral vanishes because there are no jumps for $u$ and $p$ at the crack tips.

Lemma 3.11. Let $J_{\alpha}(\Gamma)$ be the cost function (1.2), evaluated with the solution $u^{\alpha}$ of the imperfect interface model. Let $J_{*}(\Gamma)$ be the same cost function (1.2), evaluated with the solution $u^{*}$ of the perfect bonding equation (2.6). Assume that the sequences $u^{\alpha}$ and $p^{\alpha}$ are uniformly bounded in $H^{2}\left(\Omega_{1}\right)$ and $H^{2}\left(\Omega_{2}\right)$, as a goes to $+\infty$, and that the jumps of the tangential derivatives $\sqrt{\alpha}\left[\partial_{\tau} u^{\alpha}\right]$ and $\sqrt{\alpha}\left[\partial_{\tau} u^{\alpha}\right]$ are also bounded in $L^{2}(\Gamma)$. Then, for fixed $\theta \in \Theta_{a d}$ :

$$
\lim _{\alpha \rightarrow+\infty} J_{\alpha}(\Gamma)=J_{*}(\Gamma) \text { and } \lim _{\alpha \rightarrow+\infty}\left\langle J_{\alpha}^{\prime}(\Gamma), \theta\right\rangle=\left\langle J_{*}{ }^{\prime}(\Gamma), \theta\right\rangle .
$$

Remark 3.12. The assumption on the $H^{2}$-bounds for $u^{\alpha}$ and $p^{\alpha}$ is an extension of our previous Assumption 2.3. The assumption on the jumps of the tangential derivatives are of the same type as the bounds on $\sqrt{\alpha}\left[u^{\alpha}\right]$ obtained in the proof of Lemma 2.4. We believe these uniform bounds hold true for a smooth interface and smooth data. However, we are unable to find such a result in the literature since the imperfect interface model (1.1) is not so common. Conceptually, it is not very hard to imagine a proof, using standard arguments from regularity theory (partition of unity, difference quotients for translations of the solution in the case of a plane interface, etc.) but checking all the details would 
result in a lengthy proof which is not the topic of the present paper. So we prefer to state it as an assumption. Note however, that the one dimensional case is obvious to check by an explicit computation of the solution.

Proof. The first result is immediate thanks to Lemma 2.4. For the second one, using the interface conditions $A \partial_{\nu} u^{\alpha}=\alpha\left[u^{\alpha}\right], A \partial_{\nu} p^{\alpha}=\alpha\left[p^{\alpha}\right]$, the derivative of $J_{\alpha}$ is written as:

$$
\begin{aligned}
\left\langle J_{\alpha}^{\prime}(\Gamma), \theta\right\rangle=\int_{\Gamma}\left(-\left[j\left(u^{\alpha}\right)\right]\right. & \left.-\left[A \partial_{\tau} u^{\alpha} \cdot \partial_{\tau} p^{\alpha}\right]+f \frac{A \partial_{\nu} p^{\alpha}}{\alpha}+A \partial_{\nu} u^{\alpha} A \partial_{\nu} p^{\alpha}\left(\frac{\kappa}{\alpha}+\left[A^{-1}\right]+\frac{\partial_{\nu} \alpha}{\alpha^{2}}\right)\right) \theta \cdot \nu d s \\
+ & \int_{\partial \Gamma \cap \Gamma_{N}} \alpha\left[u^{\alpha}\right]\left[p^{\alpha}\right] \theta \cdot \tau d l+\int_{\partial \Gamma \cap \Gamma_{N}}\left(g_{N}\left[p^{\alpha}\right]-\left[k\left(u^{\alpha}\right)\right]\right) \theta \cdot \boldsymbol{t} d l .
\end{aligned}
$$

Using Lemma 2.4, the trace theorem, together with the regularity and quadratic growth of $j$, we have, as $\alpha \rightarrow+\infty$, that $\left[u^{\alpha}\right] \rightarrow 0$ strongly in $L^{2}(\Gamma)$ and $\left[j\left(u^{\alpha}\right)\right] \rightarrow 0$ strongly in $L^{1}(\Gamma)$. The first term in (3.10) thus vanishes in the limit. In order to deal with the other terms involving derivatives or the terms carried by the interface boundary $\partial \Gamma$, we rely on our assumption on the $H^{2}$-bounds for $u^{\alpha}$ and $p^{\alpha}$.

Because of that assumption, the sequences $u_{i}^{\alpha}$ converge to $u_{i}^{*}$ strongly in $H^{1}\left(\Omega_{i}\right)$ and weakly in $H^{2}\left(\Omega_{i}\right)$. In particular the gradients $\nabla u_{i}^{\alpha}$ converge to $\nabla u_{i}^{*}$ weakly in $H^{1}\left(\Omega_{i}\right)^{d}$ and, by the trace theorem, weakly in $H^{1 / 2}(\Gamma)^{d}$. Using the compact embedding of $H^{1 / 2}(\Gamma)$ into $L^{2}(\Gamma)$ (see [24, Theorem 3.85]) we deduce the strong convergence of the trace of the gradients in $L^{2}(\Gamma)^{d}$. Thus, $\left[A \partial_{\tau} u^{\alpha} \cdot \partial_{\tau} p^{\alpha}\right]$ converge strongly in $L^{1}(\Gamma)$ to $\left[A \partial_{\tau} u^{*} \cdot \partial_{\tau} p^{*}\right]=$ $[A] \partial_{\tau} u^{*} \cdot \partial_{\tau} p^{*}$, because the tangential derivative is continuous through the perfect interface. For the same reason, we have

$$
\lim _{\alpha \rightarrow+\infty} \int_{\Gamma}\left[A^{-1}\right] A \partial_{\nu} u^{\alpha} A \partial_{\nu} p^{\alpha} \theta \cdot \nu d s=\int_{\Gamma}\left[A^{-1}\right] A \partial_{\nu} u^{*} A \partial_{\nu} p^{*} \theta \cdot \nu d s .
$$

Furthermore, the $H^{2}$-bounds imply the following estimate:

$$
\left|\int_{\Gamma} f \frac{A \partial_{\nu} p^{\alpha}}{\alpha} \theta \cdot \nu\right| d s \leq \frac{C}{\alpha}\left\|\nabla p_{i}^{\alpha}\right\|_{L^{2}(\Gamma)^{d}} \leq \frac{C}{\alpha} \rightarrow 0 .
$$

A similar argument applies for all integrals on $\Gamma$, involving normal derivatives multiplied by negative powers of $\alpha$, which vanish in the limit.

We now pass to the limit in the two last integrals of (3.10) which are carried by $\partial \Gamma$. Since the sequences $u_{i}^{\alpha}$ converge to $u_{i}^{*}$ weakly in $H^{2}\left(\Omega_{i}\right)$, by two successive application of the trace theorem and compact embedding of $H^{1 / 2}$ into $L^{2}$ (from $\Omega_{i}$ to $\Gamma$ and from $\Gamma$ to $\partial \Gamma), u_{i}^{\alpha}$ converge to $u_{i}^{*}$ strongly in $L^{2}(\partial \Gamma)$. The same is true for the adjoint and the limit of the last integral vanishes since $u^{*}$ and $p^{*}$ are continuous through the perfect interface. For the penultimate integral, we already checked at the end of the proof of Lemma 2.4 that the jump $\sqrt{\alpha}\left[u^{\alpha}\right]$ converges strongly to 0 in $L^{2}(\Gamma)$ (a similar argument holds true for $\sqrt{\alpha}\left[p^{\alpha}\right]$ ). By assumption the jumps of the tangential derivatives $\sqrt{\alpha}\left[\partial_{\tau} u^{\alpha}\right]$ and $\sqrt{\alpha}\left[\partial_{\tau} u^{\alpha}\right]$ are bounded in $L^{2}(\Gamma)$. Thus, by the trace theorem $\sqrt{\alpha}\left[u^{\alpha}\right]$ and $\sqrt{\alpha}\left[p^{\alpha}\right]$ converge strongly to 0 in $L^{2}(\partial \Gamma)$. It implies that the penultimate integral vanishes in the limit $\alpha \rightarrow+\infty$.

Remark 3.13. From Theorem 3.7, for a surface $\Gamma$ without boundary, we can formally deduce a descent direction $\theta$ for the cost function $J$, as we have:

$$
\left\langle J^{\prime}(\Gamma), \theta\right\rangle=-\int_{\Gamma} v_{\Gamma} \theta \cdot \nu d s .
$$


So, it suffices to take the vector field $\theta(s)=v_{\Gamma}(s) \nu(s), s \in \Gamma$, which implies:

$$
\left\langle J^{\prime}(\Gamma), \theta\right\rangle=-\int_{\Gamma} v_{\Gamma}^{2} d s \leq 0
$$

at least if $v_{\Gamma}$ belongs to $L^{2}(\Gamma)$. However, this choice may not belong to $\Theta_{a d}$, as $L^{2}\left(\Gamma, \mathbb{R}^{d}\right)$ is not a subset of $W^{1, \infty}\left(D, \mathbb{R}^{d}\right)$ in general. Moreover, even ignoring this regularity issue, this choice may not be suitable due to numerical inaccuracies and lack of validity away from $\Gamma$, see ['] for a more detailed discussion.

In the next section we present a classical method to obtain a well defined descent direction by means of identifying the linear form $J^{\prime}(\Gamma) \in W^{1, \infty}\left(D, \mathbb{R}^{d}\right)^{*}$ with a gradient $\nabla J(\Gamma)$ in a such way that the duality product $\left\langle J^{\prime}(\Gamma), \theta\right\rangle$ is identified with an inner product $a(\cdot, \cdot)$ on an adequate Hilbert space $V$.

\section{The Level Set Method}

The level set method has been proposed by Osher and Sethian [46] for tracking fronts and free boundaries. It is often used in structural optimization because it allows to perform shape and topology optimization. Usually, it relies on a fixed mesh of a working domain. However, we shall not follow this usual approach since, as we are dealing with imperfect transmission conditions across the interface, we need to keep the most accurate possible track of $\Gamma$. Rather, we rely on a level set based mesh evolution method for the optimization process, following the strategy introduced in [6].

We consider a fixed computational working domain $D \subset \mathbb{R}^{d}$ in which all admissible phase subdomains $\Omega_{1}, \Omega_{2}$ are included and such that the loaded boundaries $\Gamma_{D}, \Gamma_{N}$ are included in $\partial D$. The mesh on $D$ is not fixed: we remesh it along iterations, based on a level set function $\phi$ for the internal phase $\Omega_{1}$, whose zero level set defines the interface $\Gamma$. The level set $\phi$ is defined on $D$ by:

$$
\left\{\begin{array}{l}
\phi(x)<0 \Leftrightarrow x \in \Omega_{1} \\
\phi(x)=0 \Leftrightarrow x \in \Gamma \\
\phi(x)>0 \Leftrightarrow x \in \Omega_{2}
\end{array}\right.
$$

In order to track the evolution of the interface $\Gamma$ as we perform the minimization of the cost functional, we relate the motion of the level set function $\phi$ for $\Gamma^{k-1}$ (the interface at the $(k-1)$ iteration of the numerical algorithm) and the normal velocity $\theta=-v n$ given by (3.11), which ensures that the new shape $\Gamma^{k}:=\Gamma_{t \theta}^{k-1}$ is such that $J\left(\Gamma^{k}\right)<J\left(\Gamma^{k-1}\right)$ for a descent step $t>0$. To move $\Gamma$ we solve the following linear transport equation (introducing a pseudo-time variable for the descent step $t$ ):

$$
\partial_{t} \phi+\theta \cdot \nabla \phi=0 \text { on }[0, \tau] \times D,
$$

with initial condition $\phi(0, x)$ taken as the input level set. The new level set will correspond to $\phi(\tau, x)$. For this task, we use the solver advect, which solves equation (4.2) using a numerical scheme based on the method of characteristics (see [17] for more details on this solver).

Finally, in order to solve equation (4.2) the velocity field $\theta$ is required on the whole domain $D$, instead of just its expression on $\Gamma$, as formally deduced in Remark 3.13. To 
this end, following [9] we perform an extension-regularization of the velocity, by solving the variational problem:

$$
\left\{\begin{array}{l}
\text { Find } Q \in V:=\left\{v \in H^{1}\left(D, \mathbb{R}^{d}\right), v \cdot n=0 \text { on } \partial D\right\} \text { such that: } \\
\forall v \in V, a(Q, v)=\int_{D}\left(\gamma_{r e g}^{2} \nabla Q: \nabla v+Q \cdot v\right) d x=\left\langle J^{\prime}(\Gamma), v\right\rangle
\end{array}\right.
$$

where the regularization parameter $\gamma_{r e g}>0$ is taken of the order of the minimal cell size in the mesh of $D$. Notice that this process is guaranteed to provide a descent direction. Indeed, taking $v=-Q$, we have

$$
\left\langle J^{\prime}(\Gamma),-Q\right\rangle=-\int_{D}\left(\gamma_{r e g}^{2} \nabla Q: \nabla Q+|Q|^{2}\right) d x \leq 0 .
$$

Another consequence of this extension-regularization process, as mentioned in Remark 3.13 , is that we found an Hilbert space $V$ and an inner product $a(\cdot, \cdot)$ such that the linear form $J^{\prime}(\Gamma)$ on $W^{1, \infty}\left(D, \mathbb{R}^{d}\right)$ is identified with a gradient $Q \in V$. This process is not fully satisfactory from a theoretical point of view because, here, $V$ is not a subset of $W^{1, \infty}\left(D, \mathbb{R}^{d}\right)$ and thus some extra regularity of $J^{\prime}(\Gamma)$ is required to make (4.3) well-posed. However, in numerical practice it works perfectly fine (see [7, Section 5.2] for a detailed discussion).

\section{$5 \quad$ Numerical Implementation}

We explain how to solve the systems (1.1), (3.7) and to compute the shape derivatives (3.5), (3.8) in numerial practice.

\subsection{Resolution of Direct Problems}

In the case of perfect bonding, it is completely standard to solve equations (2.6) and (3.4), which are posed in classical functional spaces, so any finite element software can do the job. However, for an imperfect interface, things become a bit more difficult due to the fact that the variational formulation (2.4) is not obvious to implement in classical finite element softwares (such as FreeFEM [35]) as there is no implementation of a discretization of the space $H_{b}^{1}$. In order to circumvent this difficulty, we propose to extend the function $u_{i}$, solely defined in $\Omega_{i}$, to a function $u_{i}^{\varepsilon}$ defined in the entire domain $D$, for some small extension parameter $\varepsilon>0$. In other words, we introduce the following approximated variational formulation, which relies on standard finite element spaces. For $\varepsilon>0$, define the spaces $V_{i}^{\varepsilon}$ for $i=1,2$ by:

$$
V_{i}^{\varepsilon}:=\left\{v^{\varepsilon} \in L^{2}(D) \text { such that } \nabla v^{\varepsilon} \in L^{2}\left(\Omega_{i}\right)\right\},
$$

and consider the variational problem:

Find $\left(u_{1}^{\varepsilon}, u_{2}^{\varepsilon}\right) \in V_{1}^{\varepsilon} \times V_{2}^{\varepsilon}$ such that, $u_{i}^{\varepsilon}=g_{D}$ on $\Gamma_{D}$ for $i=1,2$ and $\forall\left(v_{1}^{\varepsilon}, v_{2}^{\varepsilon}\right) \in V_{1}^{\varepsilon} \times V_{2}^{\varepsilon}$ such that $v_{i}^{\varepsilon}=0$ in $\Gamma_{D}$ :

$$
\begin{aligned}
& \sum_{i=1}^{2}\left(\int_{\Omega_{i}} A_{i} \nabla u_{i}^{\varepsilon} \cdot \nabla v_{i}^{\varepsilon} d x-\int_{\Omega_{i}} f v_{i}^{\varepsilon} d x\right)+\int_{\Gamma} \alpha\left(u_{2}^{\varepsilon}-u_{1}^{\varepsilon}\right)\left(v_{2}^{\varepsilon}-v_{1}^{\varepsilon}\right) d s \\
& \quad+\varepsilon\left(\int_{\Omega_{1}} u_{2}^{\varepsilon} v_{2}^{\varepsilon} d x+\int_{\Omega_{2}} u_{1}^{\varepsilon} v_{1}^{\varepsilon} d x\right)-\int_{\Gamma_{N} \cap \partial \Omega_{2}} g_{N} v_{2}^{\varepsilon} d s-\int_{\Gamma_{N} \cap \partial \Omega_{1}} g_{N} v_{1}^{\varepsilon} d s=0
\end{aligned}
$$


with a parameter $0<\varepsilon \ll 1\left(\varepsilon=10^{-5}\right.$ in our computations). Extra terms of order zero are included to make this variational formulation well posed: there exists a unique solution $\left(u_{1}^{\varepsilon}, u_{2}^{\varepsilon}\right) \in V_{1}^{\varepsilon} \times V_{2}^{\varepsilon}$ for the variational problem (5.1). Of course, only the restrictions of $u_{i}^{\varepsilon}$ in $\Omega_{i}$ are used in practice. We check that this approximation is well suited for our purposes with the following result.

Proposition 5.1. For $\varepsilon>0$, let $u_{1}^{\varepsilon}, u_{2}^{\varepsilon}$ be the solution of (5.1) and $p_{1}^{\varepsilon}, p_{2}^{\varepsilon}$ be the solution of an analogous approximate variational formulation of the adjoint system (3.7). Define $u^{\varepsilon}=u_{1}^{\varepsilon} \chi_{1}+u_{2}^{\varepsilon} \chi_{2} \in H_{b}^{1}$. Let $J_{\varepsilon}(\Gamma)$ be the objective function for this approximate problem:

$$
J_{\varepsilon}(\Gamma):=\int_{D} j\left(u^{\varepsilon}\right) d x+\int_{\Gamma_{N}} k\left(u^{\varepsilon}\right) d s .
$$

Then, $\lim _{\varepsilon \rightarrow 0} J_{\varepsilon}(\Gamma)=J(\Gamma)$ and, for any $\theta \in \Theta_{a d}, \lim _{\varepsilon \rightarrow 0}\left\langle J_{\varepsilon}^{\prime}(\Gamma), \theta\right\rangle=\left\langle J^{\prime}(\Gamma), \theta\right\rangle$.

Proof. By mimicking the proof of Theorem 3.7 it is easy to prove that $J_{\varepsilon}(\Gamma)$ is shape differentiable and to obtain the fomula

$$
\left\langle J_{\varepsilon}^{\prime}(\Gamma), \theta\right\rangle=\left(\left\langle J^{\prime}(\Gamma), \theta\right\rangle\right)^{\varepsilon}+\varepsilon \int_{\Gamma}\left(u_{1}^{\varepsilon} p_{1}^{\varepsilon}-u_{2}^{\varepsilon} p_{2}^{\varepsilon}\right) \theta \cdot \nu d s
$$

where $\left(\left\langle J^{\prime}(\Gamma), \theta\right\rangle\right)^{\varepsilon}$ is equal to formula (3.8) for $\left\langle J^{\prime}(\Gamma), \theta\right\rangle$ where $u$ and $p$ are replaced by their approximations $u^{\varepsilon}$ and $p^{\varepsilon}=p_{1}^{\varepsilon} \chi_{1}+p_{2}^{\varepsilon} \chi_{2}$.

For the sake of simplicity, we prove the convergences, as $\varepsilon$ goes to zero, when $\Gamma$ has no boundary, i.e., $\Omega_{1}$ is immersed in $\Omega_{2}$. By using Poincaré inequality and a trace theorem on $\Gamma$, it is easy to obtain the following uniform bounds, with respect to $\varepsilon>0$, for the solution $\left(u_{1}^{\varepsilon}, u_{2}^{\varepsilon}\right)$ of $(5.1)$

$$
\varepsilon^{1 / 2}\left\|u^{\varepsilon}\right\|_{L^{2}(D)}+\left\|u_{1}^{\varepsilon}\right\|_{H^{1}\left(\Omega_{1}\right)}+\left\|u_{2}^{\varepsilon}\right\|_{H^{1}\left(\Omega_{2}\right)} \leq C .
$$

Furthermore, $u^{\varepsilon} \in H_{b}^{1}$ is also the unique solution of:

$$
\left\{\begin{array}{l}
\text { Find } u^{\varepsilon} \in H_{b}^{1}, \text { such that } u^{\varepsilon}=g_{D} \text { on } \Gamma_{D} \text { and, for all } v \in H_{b, 0}^{1}, \\
\int_{\Omega_{1}} A_{1} \nabla u_{1}^{\varepsilon} \cdot \nabla v_{1}, d x+\int_{\Omega_{2}} A_{2} \nabla u_{2}^{\varepsilon} \cdot \nabla v_{2} d x+\int_{\Gamma} \alpha\left[u^{\varepsilon}\right][v] d s=\int_{D} f_{\varepsilon} v d x+\int_{\Gamma_{N}} g_{N} v d s,
\end{array}\right.
$$

with $f_{\varepsilon}=f-\varepsilon\left(u_{1}^{\varepsilon} \chi_{2}+u_{2}^{\varepsilon} \chi_{1}\right) \in L^{2}(D)$. Since $f_{\varepsilon}$ converges strongly to $f$ in $L^{2}(D)$ the sequence $\left(u^{\varepsilon}\right)_{\varepsilon}$ converges strongly, in the $H_{b}^{1}$-norm, to $u=u_{1} \chi_{1}+u_{2} \chi_{2} \in H_{b}^{1}$, solution of the variational problem (2.4). A similar result holds for the adjoint $p^{\varepsilon}=p_{1}^{\varepsilon} \chi_{1}+p_{2}^{\varepsilon} \chi_{2} \in H_{b}^{1}$, which converges in the $H_{b}^{1}$-strong sense to $p \in H_{b}^{1}$, solution of (3.7). In particular, $u^{\varepsilon}$ and $p^{\varepsilon}$ are uniformly bounded in $H_{b}^{1}$ and, by the trace theorem on $\Gamma$, we deduce:

$$
\left|\int_{\Gamma} \varepsilon u_{i}^{\varepsilon} p_{i}^{\varepsilon} \theta \cdot \nu d s\right| \leq C \varepsilon\left\|u^{\varepsilon}\right\|_{H_{b}^{1}}\left\|p^{\varepsilon}\right\|_{H_{b}^{1}} \leq C \varepsilon \rightarrow 0 .
$$

It remains to prove $\lim _{\varepsilon \rightarrow 0}\left(\left\langle J^{\prime}(\Gamma), \theta\right\rangle\right)^{\varepsilon}=\left\langle J^{\prime}(\Gamma), \theta\right\rangle$. Recall that, since $\Gamma$ and the source terms are smooth, for fixed $\varepsilon$, the solution $u^{\varepsilon}$ of (5.2) satisfies the extra regularity that $u_{i}^{\varepsilon} \in H^{2}\left(\Omega_{i}\right)$. More precisely the $H^{2}\left(\Omega_{i}\right)$-norm of $u_{i}^{\varepsilon}$ is bounded by the $L^{2}(D)$-norm of $f_{\varepsilon}$. Since the coefficients in the variational formulation (5.2) are independent of $\varepsilon$ and $f_{\varepsilon}$ converges strongly to $f$ in $L^{2}(D)$, we deduce that $u_{i}^{\varepsilon}$ converges strongly to $u_{i}$ in $H^{2}\left(\Omega_{i}\right)$. A similar argument applies to $p_{i}^{\varepsilon}$. Finally, we can pass to the limit in formula (3.8) for the shape derivative by using the trace theorem on $\Gamma$ for $u^{\varepsilon}$ and $p^{\varepsilon}$, as well as their first derivative, concluding the proof. 
Remark 5.2. Other methods have been proposed in the FEM literature to deal with systems in the form of (1.1), see for example [16, 33]. However, their implementation in finite element software is not straightforward as the resolution of (5.1).

\subsection{Remeshing to track the interface accurately: level set based mesh evolution method}

As is clear from the variational formulation of the direct problem, an accurate evaluation of the jump on $\Gamma$ is required. As already said, the so-called level set based mesh evolution method proposed in [6], and used for example in multi-physics problems as in [29], fits our needs. The MMG platform [26, 21] allows to perform the remeshing operations starting from a level set function $\phi$ such that $\Gamma$ is the zero iso-level and providing several options of interest (exact mesh of interface, minimal mesh size, maximal mesh size, graduation, etc.). We refer to [29, Chapter 6] for additional practical details.

Remark 5.3. The choice of this method over a simpler FE mesh-movement based algorithm relies on the fact that our method allows to capture the interface with any desired precision (given as a parameter to the software MMG) in a simple and effective way, without increasing substantially the computational cost of the algorithm and allowing significant shape modification, including topological changes. In contrast, mesh-movement methods are limited to small deformations and no topological changes, as been pointed in [7, Section 6.4].

\subsection{Normal and curvature computations}

Another delicate issue is the computation of the curvature, which appears in formula (3.8) of the shape derivative for an imperfect interface. We rely on the signed distance function (as proposed in [29]), for which we have $n_{1}=\nabla d_{\Omega_{1}}$ and $\kappa=\operatorname{div}\left(n_{1}\right)$ where $d_{\Omega_{1}}$ stands for the signed distance to the set $\Omega_{1}$ and $n_{1}$ for the unit normal vector (with respect to $\partial \Omega_{1}$ ). However, as the signed distance function is discretized as a $\mathbb{P}_{1}$ function, it is not possible to compute $\kappa$ directly from $n_{1}$ which is discretized as a piecewise constant function. We propose to compute the curvature $\kappa$ by solving the following variational problem:

$$
\left\{\begin{array}{l}
\text { Find } \kappa \in H^{1}(D), \text { such that, for all } w \in H^{1}(D) \\
\int_{D}\left(\gamma^{2} \nabla \kappa \cdot \nabla w+\kappa w\right) d x=\int_{D} \operatorname{div}\left(n_{r e g}\right) w d x
\end{array}\right.
$$

where the normal $n_{\text {reg }}$ is computed via a similar $H^{1}(D)$ regularization with source term $\nabla d_{\Omega_{1}}$. Upon discretization, $d_{\Omega_{1}}$ is a $\mathbb{P}_{1}$ function, $\nabla d_{\Omega_{1}}$ is a $\mathbb{P}_{0}$ function, while $n_{\text {reg }}$ is a $\mathbb{P}_{1}$ function, its divergence a $\mathbb{P}_{0}$ function and $\kappa$ again a $\mathbb{P}_{1}$ function.

\subsection{Constraints: Simple and Augmented Lagrangian}

As we have mentioned before, if we perform the minimization of $J$ without any additional requirement, the optimization algorithm will often fill the whole computational domain $D$ with the 'best' material (with respect to the functional $J$ ). Therefore, we take into account a volume constraint on phase $\Omega_{1}$. There are, at least, two alternatives to prescribe a constraint in an optimization problem. 
The simplest one is to introduce a Lagrangian, given by

$$
\mathcal{L}\left(\Omega_{1}\right):=J(\Gamma)+\ell\left(\int_{\Omega_{1}} d x-V_{\text {tar }}\right),
$$

where $\ell$ is a Lagrange multiplier which is updated during the optimization process in order to satisfy the constraint at convergence. This is the simplest method but its convergence is rather slow.

On the other hand, the Augmented Lagrangian method allows to actually impose a series of constraints during the optimization process, it is based on considering a merit function to be optimized. For example, if we want to impose $n$ equality constraints, given by the equations $c_{i}\left(\Omega_{1}\right)=0$, we take:

$$
\mathcal{L}(\Gamma, \lambda, \mu):=J(\Gamma)-\sum_{i=1}^{n} \lambda_{i} c_{i}\left(\Omega_{1}\right)+\sum_{i=1}^{n} \frac{\mu_{i}}{2} c_{i}^{2}\left(\Omega_{1}\right),
$$

where each $\lambda_{i}$ is expected to converge to the Lagrange multipliers of each constraint and $\mu_{i}>$ is a penalty parameter for each constraint. In order to do this, the theory (see [45]), suggest to update $\lambda_{i}^{k+1}$ as:

$$
\lambda_{i}^{k+1}=\lambda_{i}^{k}-\mu_{i}^{k} c_{i}\left(\Omega_{1}\right)
$$

is important to notice that, in this case, it is not necessary to take a sequence of $\mu_{i}^{k}$ such that $\mu_{i}^{k} \rightarrow+\infty$. The general algorithm used in our simulations is illustrated in Algorithm 1 .

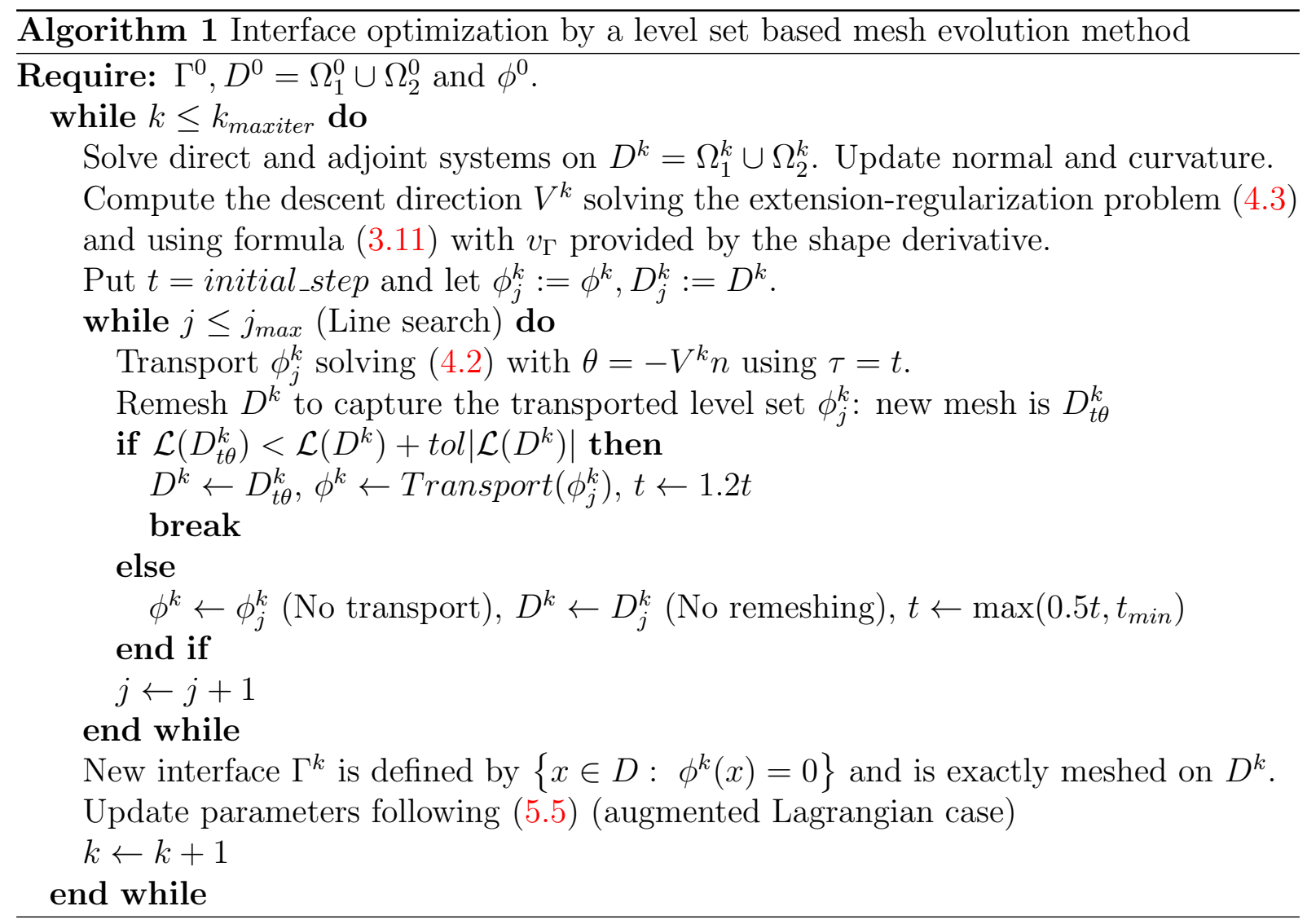




\section{$6 \quad$ Numerical Examples}

All the simulations were performed on a personal laptop with an Intel i7 8th-gen processor, $16 \mathrm{~GB}$ of RAM and SSD storage; all the computations were sequential. The finite element analysis is performed with the FreeFEM package [35], the advection step of the level set is performed using the advect tool [17] from the ISCDtoolbox, the computation of the signed distance function to a given domain is done with the mshdist tool also from the ISCDtoolbox and, finally, the remeshing steps are performed using the MMG platform $[26,21]$. In the following examples, when two materials with different conductivities are considered, we depict the weaker material in gray, and the stronger material in white. To assess the impact of the imperfect interface on the optimization process we systematically make a comparison between the perfect and imperfect interface cases on all the examples (except for Example 3, which has no perfect setting). The implementation remains the same for perfect interface cases (following Algorithm 1).

\subsection{Example 1: The Radiator}

Consider a square domain $D=(-0.5,0.5)^{2}$. There is no bulk source term, $f=0$, but an incoming unit heat flux $g_{N}=1$ on the upper side $\Gamma_{N}=[-0.5,0.5] \times\{0.5\}$, with an homogeneous Dirichlet boundary condition $g_{D}=0$ at $\Gamma_{D}=[-0.5,-0.4] \times\{-0.5\} \cup$ $[0.4,0.5] \times\{-0.5\}$ and adiabatic walls on $\partial D \backslash\left(\Gamma_{D} \cup \Gamma_{N}\right)$.
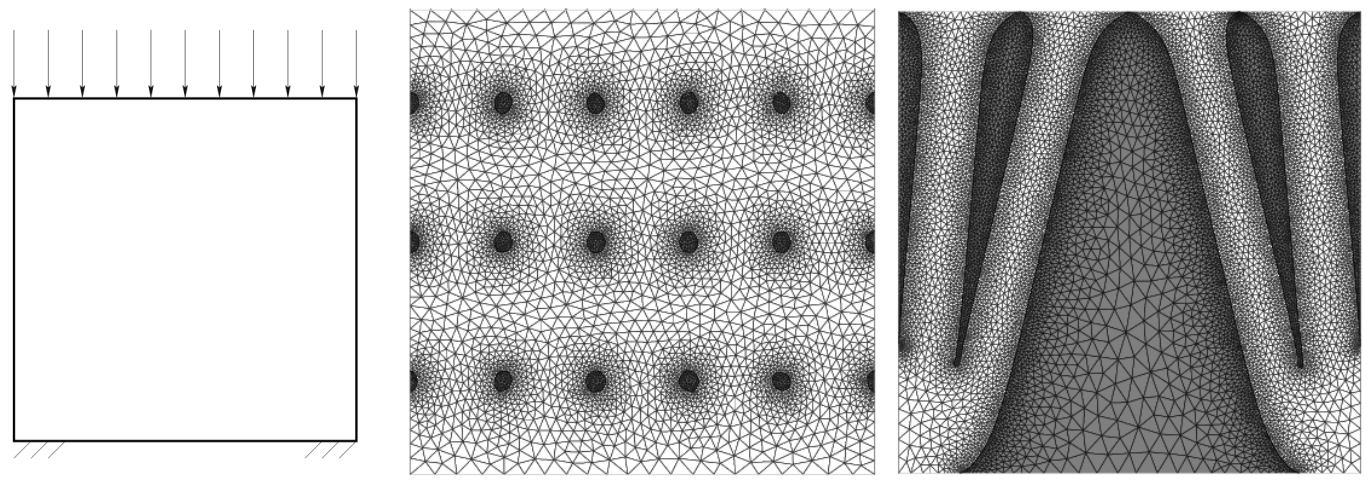

Figure 2: Radiator setting, initial and final distribution for Example 1 - Perfect interface

We have two different materials with conductivities $A_{1}=0.01$ and $A_{2}=1.0$, the aim is to determine the distribution of the materials which, for a given volume, minimizes the thermal compliance. So, we consider:

$$
J(\Gamma)=\int_{\Gamma_{N}} u d s
$$

i.e. $j(u)=0, k(u)=u$. This is a self-adjoint problem, with $p=-u$. The temperature $u$ solves (2.6) in the case of a perfect interface or (1.1) for an imperfect interface. In this example we consider $V_{t a r}=0.5$ and the initialization is the same for the two cases (see Figure 2). 

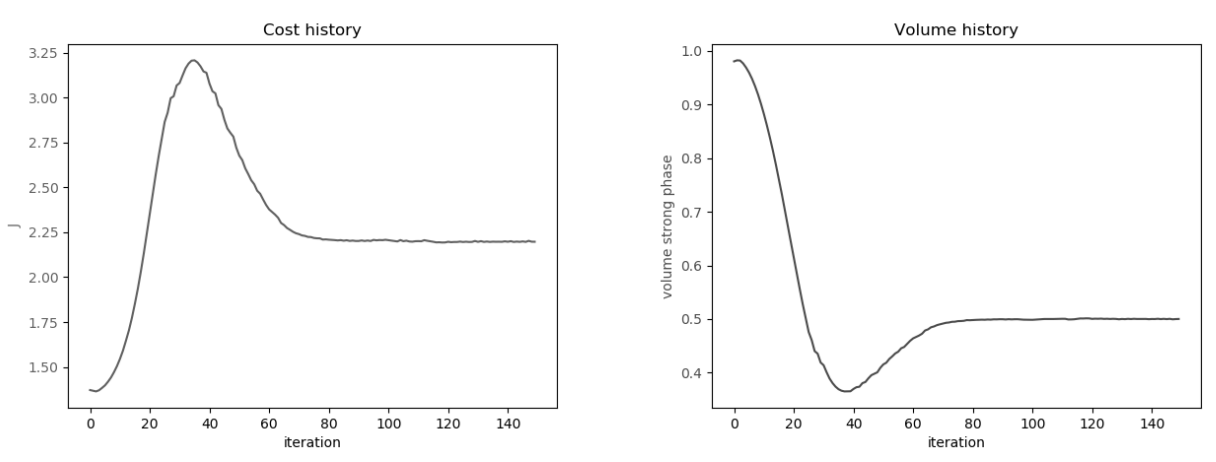

Figure 3: Convergence history for Figure 2
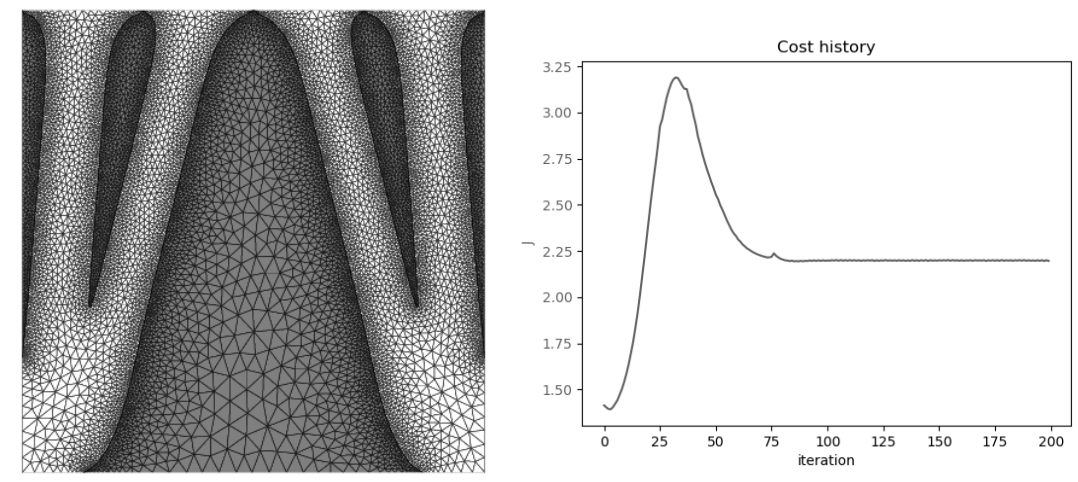

Figure 4: Final distribution and convergence history for Example 1 - Imperfect interface with $\alpha=1$

For this example, we compare optimal designs obtained with a perfect interface (Figure 2) and with an imperfect interface (Figure 4). Although we plot only the result for $\alpha=1$, we performed many other tests with different values of $\alpha$ and they all give very similar designs. It is not surprising that for different $\alpha$ the obtained configuration is essentially the same as for the perfect interface. The interface acts basically as an isolating barrier: the smaller $\alpha$, the stronger the isolation. But, since the phase $A_{1}$ is already a poor conductor, isolating it from $A_{2}$ does not add much to its weak behavior. Thus the obtained optimal configurations are all very similar. Notice also that it is reasonable for the weak phase to avoid the region of the heat source: the isolation imposed by the interface will not allow to let it go through the cold end. We will see an application of this idea on Example 4.

As can be checked on Figures 3 and 4 the optimization convergence is smooth and takes roughly 100 iterations. The final mesh has 21,219 elements for Figure 2 and 20,154 elements for Figure 4.

\subsection{Example 2: Optimal heat conduction}

Consider the following configuration (see $[29,44]$ for the original setting): we have a square domain $D=(0,1)^{2}$ heated with an uniform bulk source $f=10$, with a cold end with homogeneous Dirichlet boundary condition on $\Gamma_{D}=[0.45,0.55] \times\{0\}$ and adiabatic walls on $\partial D \backslash \Gamma_{D}$. We have in this region two different materials with conductivities $A_{1}=1.0$ 
and $A_{2}=0.001$, we want to determine the distribution of the materials which, for a given volume on the strong material $\left(V_{1}=0.2\right)$, minimizes the average temperature. So, in this context we have: $g_{D}=0, f=10$ and

$$
J(\Gamma)=\int_{\Omega_{1}} u_{1} d x+\int_{\Omega_{1}} u_{2} d x
$$

i.e. $j(u)=u, k(u)=0$. This problem is also self-adjoint, with $p=-u / f$.
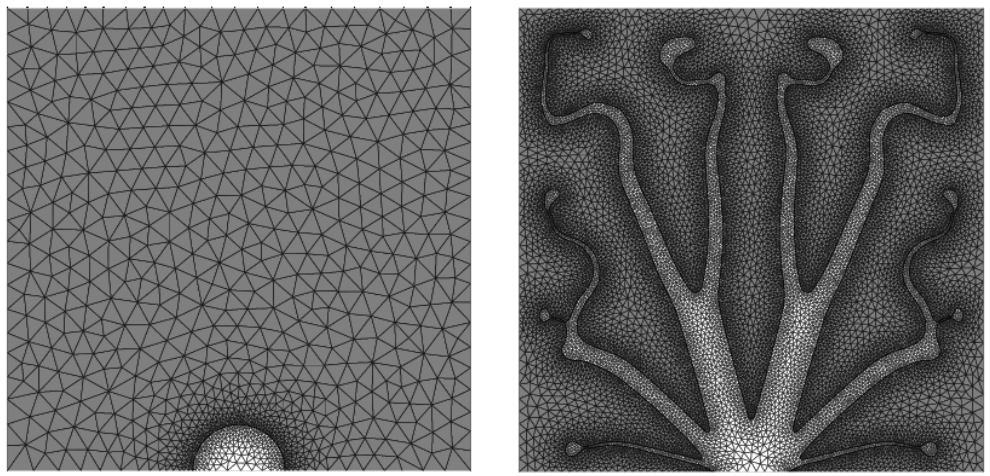

Figure 5: Initial and final distribution for Example 2 - Perfect interface
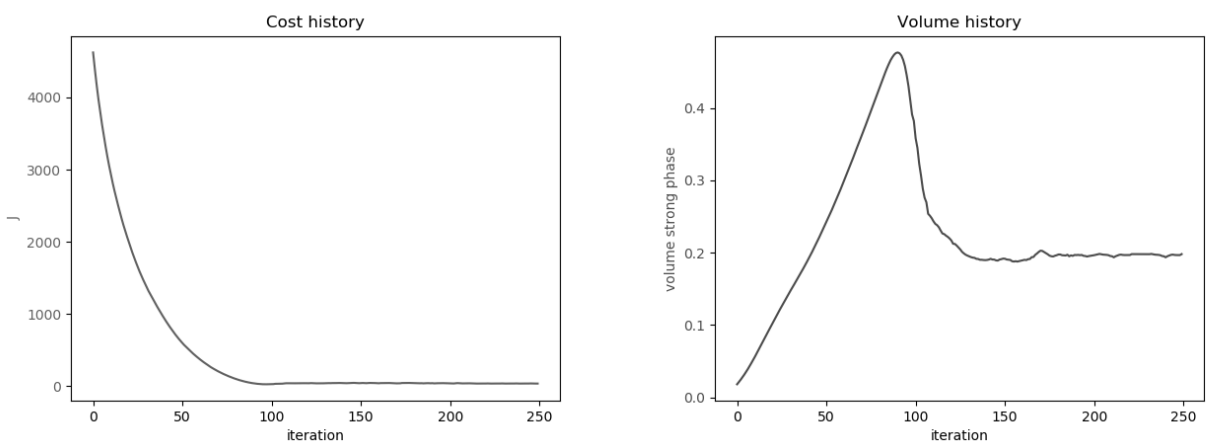

Figure 6: Convergence history for Figure 5
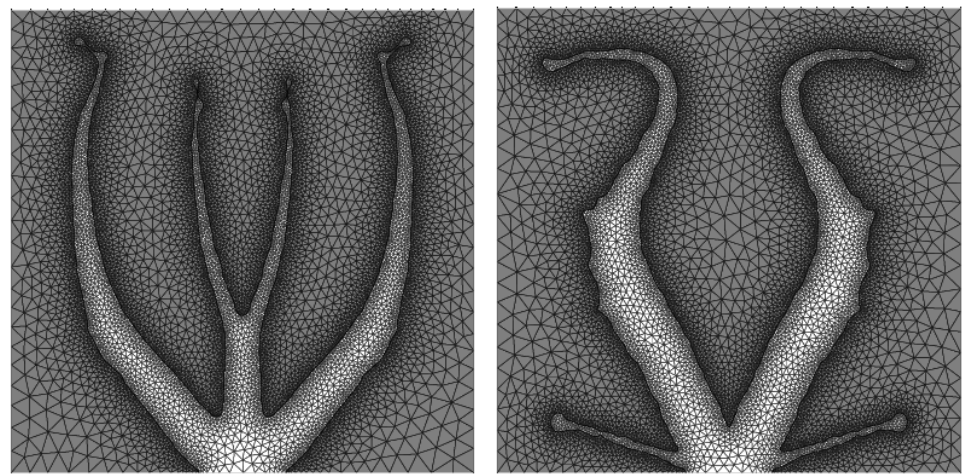

Figure 7: Final distribution for Example 2 - Imperfect interface with $\alpha=1.0$ (left), $\alpha=0.1$ (right) 


\begin{tabular}{|l|l|l|l|}
\hline Test Case & Initial Compliance & Final Compliance & Perimeter of $\Gamma$ \\
\hline Perfect Interface & 4808.91 & 37.86 & 12.13 \\
\hline Imperfect $\alpha=1.0$ & 4903.89 & 89.77 & 6.66 \\
\hline Imperfect $\alpha=0.1$ & 5183.36 & 116.03 & 5.97 \\
\hline
\end{tabular}

Table 1: Obtained compliance on each test for Example 2

The goal of this example is to show that the value of the interface conductance $\alpha$ may have a strong influence on the optimal design. We compare the optimal design for the perfect interface (Figure 5) with two values of the imperfect interface (Figure 7). Clearly, as $\alpha$ increases (meaning an improvement of the heat transmission through the interface), the design becomes more and more complex with many small branches, as well as its perimeter increases (see Table 1). For finite values of $\alpha$ there is a competition between the complexity of $\Omega_{1}$, which allows to extract efficiently the heat produced by the bulk source $f$, and the length of the interface $\Gamma$ which is a thermal barrier.

The final mesh has 29,027 elements for Figure 5 (right), 18,794 elements for Figure 7 (left) and 17,736 elements for Figure 7 (right). The convergence is smooth as can be checked on Figure 6.

\subsection{Example 3: Maximization of the compliance - The impact of the imperfect interface}

The domain is $D=(0,1)^{2}$ filled with a single phase with a unit conductivity $A_{1}=A_{2}=1$, heated with an uniform bulk source $f=1$ with homogeneous Dirichlet boundary condtions on $\partial D$. Since the two phases are identical, the interface $\Gamma$ is truly the only design variable. Its conductance is taken as $\alpha=1$. The goal is to maximize the thermal compliance:

$$
J(\Gamma):=\int_{\Omega_{1}} u_{1} d x+\int_{\Omega_{2}} u_{2} d x
$$

Of course, the longer the imperfect interface and the closer to the boundary, the larger the compliance. Nevertheless, the initialization matters and different optimal designs are obtained when varying the initial interface. Notice that in this example, the problem is again self-adjoint with $p=u$.
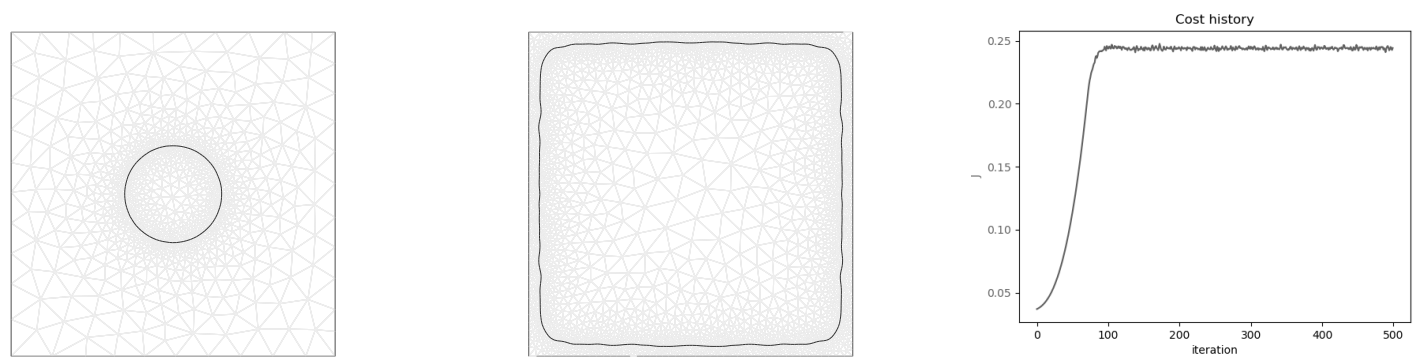

Figure 8: Initial, final distribution and convergence history for Example 3 

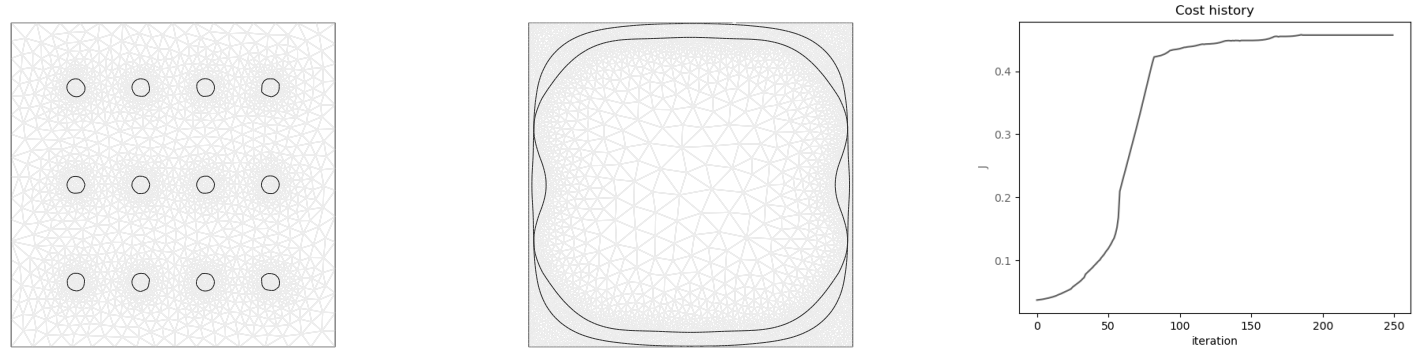

Figure 9: Another initial, final distribution and convergence history for Example 3

With two different initializations two different optimal interfaces are obtained in Figures 8 and 9 . In both cases, the imperfect interface is very close to the exterior boundary since it acts as an isolating barrier, separating the interior, where the heat source is applied, from the boundary $\partial D$, where heat can be evacuated. The compliance is almost doubled in Figure 9 compared to Figure 8, with values 0.244 and 0.451 on each case. The length of the interface is clearly important as can be checked in Figure 10 where $\Gamma$ is initialized as 8 concentric circles. Notice that the obtained value for the compliance is higher than the previously seen cases: 0.508 . Also, it is worth to notice that the components of $\Gamma$ tend to approach themselves, until there is no more room (the minimal resolution for the mesh is the lilmitation) and we cannot improve the value of $J$ anymore. The final mesh has 4,800 elements in the case of Figure 8, 6,717 elements in the case of Figure 9 and 31,264 elements in the case of Figure 10.
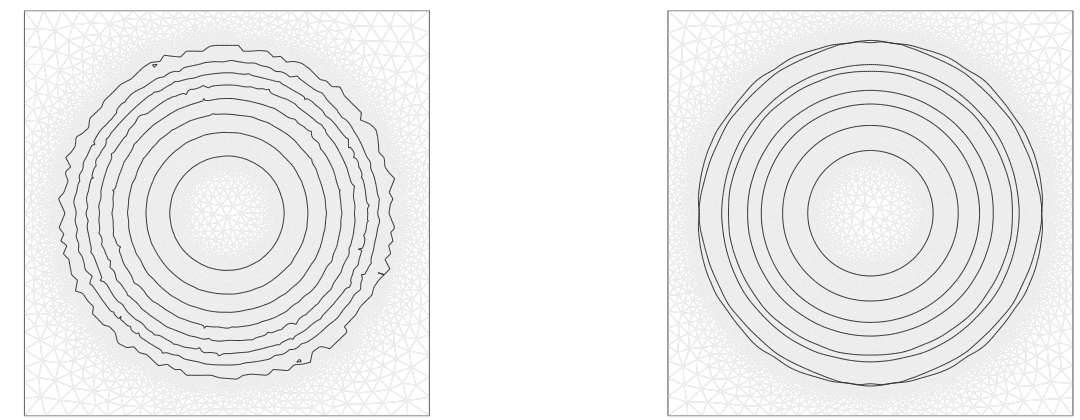

Figure 10: Initial and final distribution for Example 3

\subsection{Example 4: A quadratic cost.}

The goal of the this example is to provide a numerical test for a non self-adjoint problem. The domain $D$ is still a square $D=(-0.5,0.5)^{2}$, with a unit flux $\left(g_{N}=1\right)$ imposed on the top boundaries $\Gamma_{N}=[-0.5,0.5] \times\{0.5\}$, a homogeneous Dirichlet boundary condition $g_{D}=0$ (cold end) on $\Gamma_{D}=\{0.5\} \times[-0.2,0.2]$ and adiabatic walls $\left(A \partial_{n} u=0\right)$ on the rest of the boundary $\partial D \backslash\left(\Gamma_{D} \cup \Gamma_{N}\right)$. The domain $D$ is filled with two phases $A_{1}=1.0$ and $A_{2}=0.5$ (with a relatively low contrast). To define the objective function, we introduce a measuring region $\Gamma_{m} \subset \partial D$ which is $\Gamma_{m}=\{-0.5\} \times[-0.5,-0.2]$. The goal is to minimize the quadratic cost:

$$
J(\Gamma):=\int_{\Gamma_{m}} u(\Gamma)^{2} d s,
$$


which yields a non self-adjoint optimization problem. The corresponding adjoint system is defined by:

$$
\begin{cases}-\operatorname{div}\left(A \nabla p_{i}\right)=0 & \text { in } \Omega_{i}, \\ A \partial_{\nu} p=\alpha\left(p_{2}-p_{1}\right) & \text { on } \Gamma, \\ p=0 & \text { on } \Gamma_{D}, \\ A \partial_{n} p=0 & \text { on } \Gamma_{N} \backslash \Gamma_{m}, \\ A \partial_{n} p=-2 u & \text { on } \Gamma_{m} .\end{cases}
$$

Notice that in this example there is imposed volume constraint.

The idea is to see if, as one can expect, the interface moves in order to use the weak material as a barrier, pushing the strong phase near the cold end, letting the temperature flux go out and avoiding to go further inside the domain in order to minimize the temperature in the measuring region.
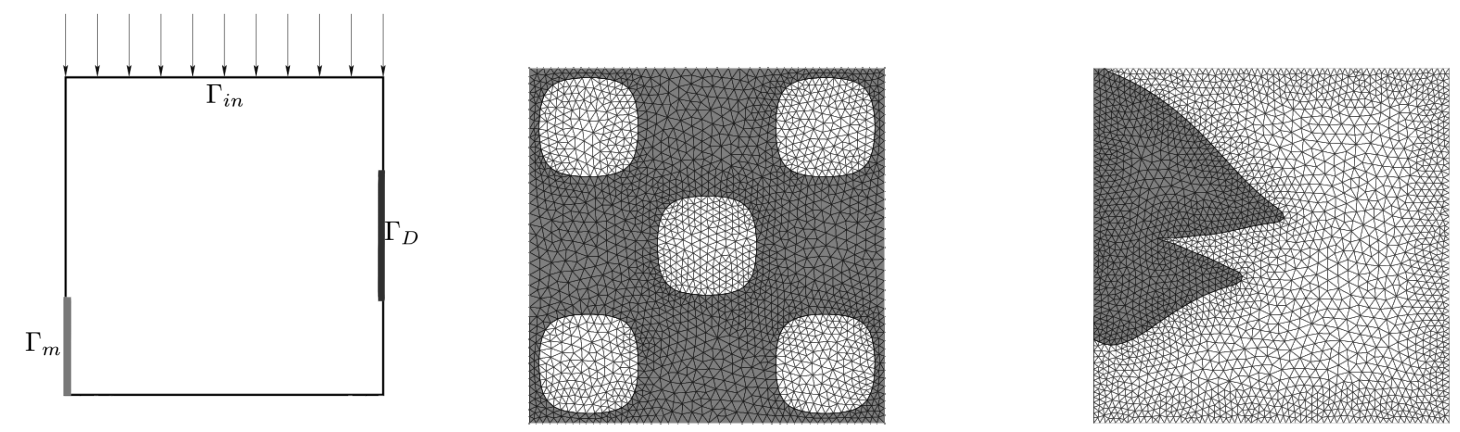

Figure 11: Setting, initial and final distribution for Example 4 - Perfect interface
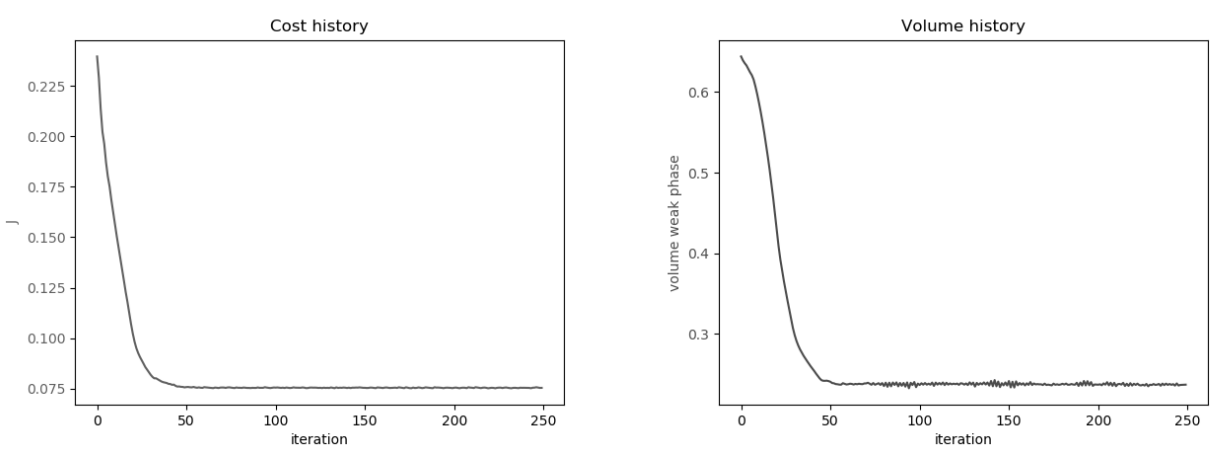

Figure 12: Convergence history for Figure 11 

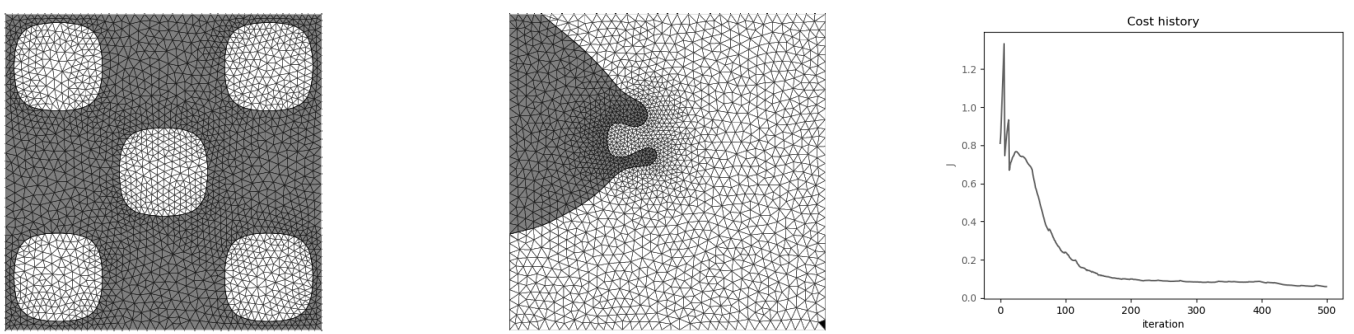

Figure 13: Initial and final distribution for Example 4 - Imperfect interface with $\alpha=1$

As can be seen for the two cases of a perfect interface (Figure 11) and of an imperfect interface (Figure 13), the weaker material distributes in such a way that it prevents the heat to approach directly the measuring zone $\Gamma_{m}$. As could be expected, the imperfect interface, acting as a thermal barrier, leads to a final value of the cost $J$ which is smaller (0.0599) than for a perfect interface (0.0754). However, the convergence is faster for the perfect interface, as can be checked on Figure 12. The final mesh has 4,725 elements for Figure 11 right, and 3,428 elements for Figure 13 middle.

\subsection{Example 5: Least Square Inverse Problem.}

This example is a toy model of an inverse problem. The goal is to reconstruct the interface, knowing the conductivity of the two phases and with some given synthetic data in the full domain. We do not claim that it is realistic inverse problem but it is a good numerical test for our optimization method. Recall, as inverse theory asserts, that domain reconstruction is an ill posed problem. The setting is that of the radiator example in Subsection 6.1 (with the same source term and boundary conditions), except that the the two phases have a low contrast with known conductivities $A_{1}=1.0$ and $A_{2}=0.5$. The problem is to determine the target interface $\Gamma^{*}$, displayed on Figure 14 (right). (Note in passing that $\Gamma^{*}$ is the result of a compliance minimization for a perfect interface.) The only known information about $\Gamma^{*}$ is the synthetic data $u_{\text {data }}$ which is the associated temperature computed by solving one of the model (2.6) or (1.1). In order to recover the interface, we minimize the least square type cost:

$$
J(\Gamma):=\int_{D}\left|u(\Gamma)-u_{d a t a}\right|^{2} d x
$$

where $u(\Gamma)$ solves the state equation on $D$. In all following cases, the optimization problem is initialized with the interface $\Gamma^{0}$ displayed on Figure 14 (left). We do not impose any constraint on the minimization of $J$. We perform three numerical tests. 

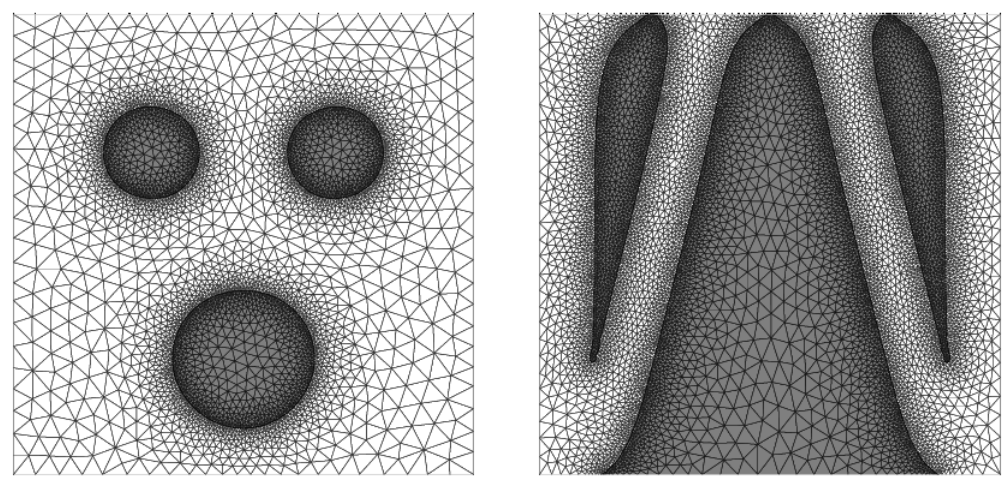

Figure 14: Initialization $\Gamma^{0}$ (left) and target interface $\Gamma^{*}$ (right) for Example 5

A first test amounts to use a perfect interface to compute the data $u_{\text {data }}$ and the solution $u(\Gamma)$. In other words, they are both solution of equation (2.6). The minimization of $J(\Gamma)$ involves an adjoint equation given by (3.4). The results are depicted in Figure 15. It is somehow a reference computation and one can check that the reconstruction is not very good although the objective function is almost zero.
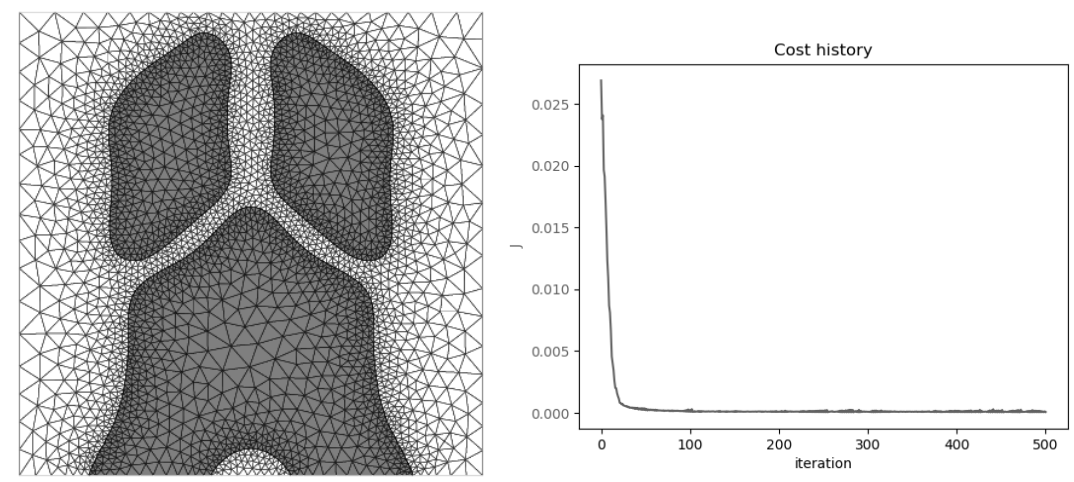

Figure 15: Obtained distribution and convergence history for test 1 of Example 5 - Perfect interface

A second test amounts to still consider $u_{\text {data }}$ as the solution of system (2.6) (perfect interface), but now $u(\Gamma)$ solves the state equation (1.1) (imperfect interface) with $\alpha=1$. The minimization of $J(\Gamma)$ involves a different adjoint equation given by (3.7). The results are depicted in Figure 16 and, of course, they are worse than for the first test because there is a mismatch between the two models used for computing the synthetic data on the one hand and for optimizing on the other hand. 

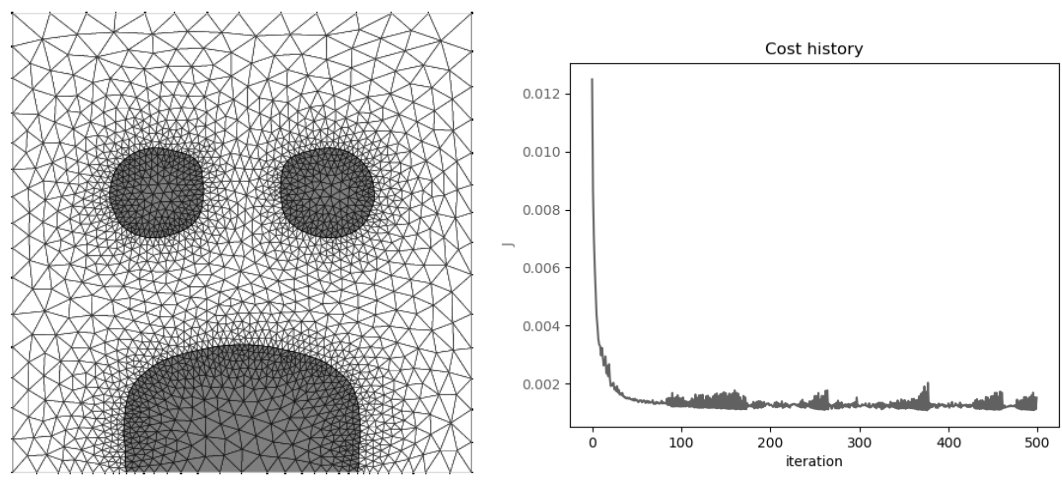

Figure 16: Obtained distribution and convergence history for test 2 of Example 5 - Imperfect interface with $\alpha=1$

A third test is concerned with both $u_{\text {data }}$ and $u(\Gamma)$ given by solving equation (1.1) (imperfect interface) with $\alpha=1$. The results are depicted in Figure 17 and the reconstruction of $\Gamma^{*}$ is much better than in the previous cases although the value of the objective function is not closer to zero. The reason for this qualitatively better result is that, since the two phases do not have a large contrast, it is easier to recognize an imperfect interface than a perfect one.

The final distribution mesh has 5,680 elements in the case of Figure 15, 3,848 elements in the case of Figure 16 and 6,300 elements in the case of Figure 17. In all three tests the convergence is rather smooth and takes place after roughly 100 iterations.
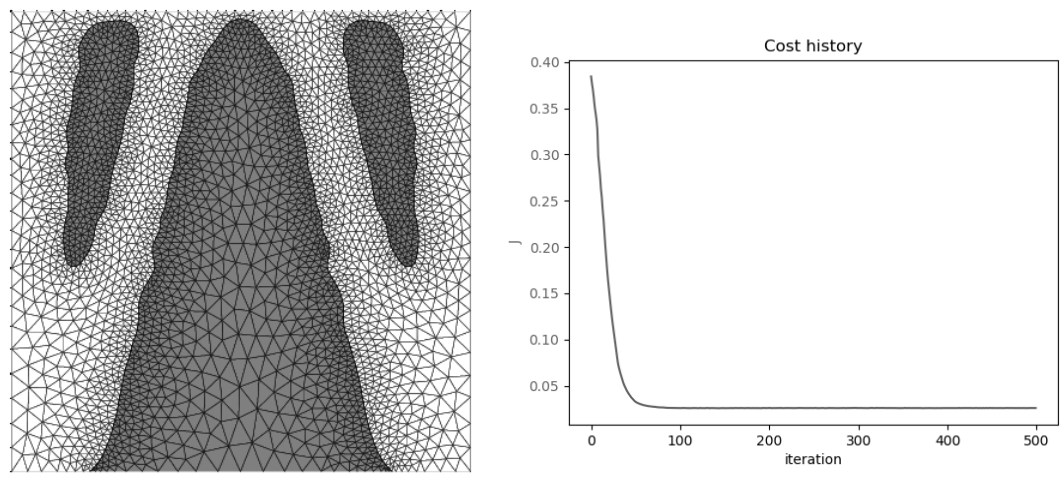

Figure 17: Obtained distribution and convergence history for test 3 of Example 5 - Imperfect interface with $\alpha=1$

\section{Conclusion}

The main objective of this article is to perform shape optimization on a steady-state heat diffusion system when the design variable is an imperfect interface. For this goal, the shape derivative of a general class of cost functionals was obtained and a gradient-descent algorithm was successfully implemented in the level set setting. One key feature of our approach is the use of a remeshing algorithm such that the interface is exactly meshed at each optimization iteration, thus minimizing the numerical errors in the treatment of the imperfect interface. Several numerical experiments have been performed, motivated 
by optimal design or inverse problems. In all cases our algorithm was able to significantly decrease the objective function, converging to a local minimum. From these experiences we observed that the influence of the imperfect interface can be significative, from the point of view of the obtained design and/or the obtained cost value. However, is some cases, in particular when the contrast between the phases is high, there is no noticeable differences between the optimized designs with a perfect or an imperfect interface. We plan to extend our approach to the linearized elasticity setting with an application to the optimization of support structures in additive manufacturing. Indeed, to ease the removal of these supports, they are often weakly connected to the main structure by means of a jagged or indented layer, which can be modeled, at first order, as an imperfect interface between the supports and the built structure. Therefore, our approach does make sense although it may be possible to enrich the model of elastic imperfect interface by adding a contact or non-interpenetrating condition.

\section{Acknowledgements}

The authors thank M. Boissier, A. Ferrer and F. Feppon for their useful comments and fruitful discussions. This work was supported by the SOFIA project, funded by BPI (Banque Publique d'Investissement).

\section{References}

[1] J. Aboudi. Mechanics of composite materials: a unified micromechanical approach, volume 29 of Studies in Applied Mechanics. Elsevier Scientific Publishing Co., Amsterdam, 1991.

[2] G. Allaire. Shape optimization by the homogenization method, volume 146 of Applied Mathematical Sciences. Springer-Verlag, New York, 2002.

[3] G. Allaire. Conception optimale de structures, volume 58 of Mathématiques \& Applications (Berlin) [Mathematics \& Applications]. Springer-Verlag, Berlin, 2007. With the collaboration of Marc Schoenauer (INRIA) in the writing of Chapter 8.

[4] G. Allaire and B. Bogosel. Optimizing supports for additive manufacturing. Struct. Multidiscip. Optim., 58(6):2493-2515, 2018.

[5] G. Allaire, C. Dapogny, G. Delgado, and G. Michailidis. Multi-phase structural optimization via a level set method. ESAIM Control Optim. Calc. Var., 20(2):576$611,2014$.

[6] G. Allaire, C. Dapogny, and P. Frey. Shape optimization with a level set based mesh evolution method. Comput. Methods Appl. Mech. Engrg., 282:22-53, 2014.

[7] G. Allaire, C. Dapogny, and F. Jouve. Shape and topology optimization. In A. Bonito and R. H. Nochetto, editors, Geometric Partial Differential Equations - Part II, volume 22 of Handbook of Numerical Analysis. Elsevier, 2021.

[8] G. Allaire and L. Jakabčin. Taking into account thermal residual stresses in topology optimization of structures built by additive manufacturing. Math. Models Methods Appl. Sci., 28(12):2313-2366, 2018.

[9] G. Allaire, F. Jouve, and A.-M. Toader. A level-set method for shape optimization. C. R. Math. Acad. Sci. Paris, 334(12):1125-1130, 2002. 
[10] G. Allaire, F. Jouve, and N. Van Goethem. Damage and fracture evolution in brittle materials by shape optimization methods. J. Comput. Phys., 230(12):5010-5044, 2011.

[11] L. Ambrosio and G. Buttazzo. An optimal design problem with perimeter penalization. Calc. Var. Partial Differential Equations, 1(1):55-69, 1993.

[12] C. Barlier and A. Bernard. Fabrication additive - Du Prototypage Rapide à l'impression 3D. Dunod, Paris, 2016.

[13] M. P. Bendsøe and O. Sigmund. Topology optimization. Springer-Verlag, Berlin, 2003. Theory, methods and applications.

[14] C. Bernardi and O. Pironneau. Sensitivity of Darcy's law to discontinuities. Chinese Ann. Math. Ser. B, 24(2):205-214, 2003.

[15] M. M. Brahim. Méthodes d'éléments finis pour le problème de changement de phase en milieux composites. PhD thesis, Bordeaux, 2016.

[16] J. Bramble and J. King. A finite element method for interface problems in domains with smooth boundaries and interfaces. Adv. Comput. Math., 6(2):109-138 (1997), 1996.

[17] C. Bui, C. Dapogny, and P. Frey. An accurate anisotropic adaptation method for solving the level set advection equation. Internat. J. Numer. Methods Fluids, 70(7):899922, 2012 .

[18] J. Céa. Conception optimale ou identification de formes: calcul rapide de la dérivée directionnelle de la fonction coût. RAIRO Modél. Math. Anal. Numér., 20(3):371402, 1986.

[19] A. Chambolle. A density result in two-dimensional linearized elasticity, and applications. Arch. Ration. Mech. Anal., 167(3):211-233, 2003.

[20] D. Chenais. On the existence of a solution in a domain identification problem. $J$. Math. Anal. Appl., 52(2):189-219, 1975.

[21] C. Dapogny, C. Dobrzynski, and P. Frey. Three-dimensional adaptive domain remeshing, implicit domain meshing, and applications to free and moving boundary problems. J. Comput. Phys., 262:358-378, 2014.

[22] C. Dapogny, N. Lebbe, and E. Oudet. Optimization of the shape of regions supporting boundary conditions. Numer. Math., 146(1):51-104, 2020.

[23] M. C. Delfour and J.-P. Zolésio. Shapes and geometries, volume 22 of Advances in Design and Control. Society for Industrial and Applied Mathematics (SIAM), Philadelphia, PA, second edition, 2011. Metrics, analysis, differential calculus, and optimization.

[24] F. Demengel and G. Demengel. Functional spaces for the theory of elliptic partial differential equations. Universitext. Springer, London; EDP Sciences, Les Ulis, 2012. Translated from the 2007 French original by Reinie Erné. 
[25] D. A. Di Pietro and A. Ern. Mathematical aspects of discontinuous Galerkin methods, volume 69 of Mathématiques \&6 Applications (Berlin) [Mathematics \&6 Applications]. Springer, Heidelberg, 2012.

[26] C. Dobrzynski and P. Frey. Anisotropic delaunay mesh adaptation for unsteady simulations. In Proceedings of the 17th international Meshing Roundtable, pages 177-194. Springer, 2008.

[27] V. Dolejší and M. Feistauer. Discontinuous Galerkin method, volume 48 of Springer Series in Computational Mathematics. Springer, Cham, 2015. Analysis and applications to compressible flow.

[28] P. Duysinx, L. Van Miegroet, T. Jacobs, and C. Fleury. Generalized shape optimization using x-fem and level set methods. In IUTAM Symposium on Topological Design Optimization of Structures, Machines and Materials, pages 23-32. Springer, 2006.

[29] F. Feppon. Shape and topology optimization of multiphysics systems. PhD thesis, Paris Saclay, 2019.

[30] G. Fremiot and J. Sokolowski. Hadamard formula in nonsmooth domains and applications. In Partial differential equations on multistructures. Proceedings of the conference, Luminy, France, pages 99-120. New York, NY: Marcel Dekker, 2001.

[31] M. Gan and C. Wong. Practical support structures for selective laser melting. Journal of Materials Processing Technology, 238:474 - 484, 2016.

[32] I. Gibson, D. Rosen, and B. Stucker. Additive Manufacturing Technologies. Springer New York, 2015.

[33] A. Hansbo and P. Hansbo. An unfitted finite element method, based on Nitsche's method, for elliptic interface problems. Comput. Methods Appl. Mech. Engrg., 191(4748):5537-5552, 2002.

[34] J. Haslinger and J. Dvořák. Optimum composite material design. RAIRO Modél. Math. Anal. Numér., 29(6):657-686, 1995.

[35] F. Hecht. New development in freefem++. J. Numer. Math., 20(3-4):251-265, 2012.

[36] A. Henrot and M. Pierre. Variation et optimisation de formes, volume 48 of Mathématiques \& Applications (Berlin) [Mathematics \& Applications]. Springer, Berlin, 2005. Une analyse géométrique. [A geometric analysis].

[37] F. Hettlich and W. Rundell. The determination of a discontinuity in a conductivity from a single boundary measurement. Inverse Problems, 14(1):67-82, 1998.

[38] A. L. Karchevsky. Reconstruction of pressure velocities and boundaries of thin layers in thinly-stratified layers. J. Inverse Ill-Posed Probl., 18(4):371-388, 2010.

[39] S. Lang. Fundamentals of differential geometry, volume 191 of Graduate Texts in Mathematics. Springer-Verlag, New York, 1999.

[40] F. Lene and D. Leguillon. Study of the influence of slip between the constituents of a composite material on its effective behavior coefficients. Journal de Mecanique, 20(3):509-536, 1981. 
[41] G. Leugering, J. Sokołowski, and A. Żochowski. Control of crack propagation by shape-topological optimization. Discrete Contin. Dyn. Syst., 35(6):2625-2657, 2015.

[42] R. Lipton. Optimal fiber configurations for maximum torsional rigidity. Arch. Rational Mech. Anal., 144(1):79-106, 1998.

[43] R. Lipton. Reinforcement of elastic structures in the presence of imperfect bonding. Quart. Appl. Math., 59(2):353-364, 2001.

[44] G. Marck and Y. Privat. On some shape and topology optimization problems in conductive and convective heat transfers. 2014.

[45] J. Nocedal and S. J. Wright. Numerical optimization. Springer Series in Operations Research and Financial Engineering. Springer, New York, second edition, 2006.

[46] S. Osher and J. A. Sethian. Fronts propagating with curvature-dependent speed: algorithms based on Hamilton-Jacobi formulations. J. Comput. Phys., 79(1):12-49, 1988.

[47] O. Pantz. Sensibilité de l'équation de la chaleur aux sauts de conductivité. $C$. $R$. Math. Acad. Sci. Paris, 341(5):333-337, 2005.

[48] K. Pietrak and T. S. Wiśniewski. A review of models for effective thermal conductivity of composite materials. Journal of Power Technologies, 95(1):14-24, 2014.

[49] L. Tartar. The general theory of homogenization, volume 7 of Lecture Notes of the Unione Matematica Italiana. Springer-Verlag, Berlin; UMI, Bologna, 2009. A personalized introduction.

[50] N. Vermaak, G. Michailidis, G. Parry, R. Estevez, G. Allaire, and Y. Bréchet. Material interface effects on the topology optimization of multi-phase structures using a level set method. Struct. Multidiscip. Optim., 50(4):623-644, 2014.

[51] L. Yin and G. Ananthasuresh. Topology optimization of compliant mechanisms with multiple materials using a peak function material interpolation scheme. Structural and Multidisciplinary Optimization, 23(1):49-62, 2001. 